\title{
Seventy Five (Thousand) Unsolved Problems in Analysis and Partial Differential Equations
}

\author{
Vladimir Maz'ya
}

\begin{abstract}
This is a collection of open problems concerning various areas in function theory, functional analysis, theory of linear and nonlinear partial differential equations.
\end{abstract}

Mathematics Subject Classification. Primary: 46E35, 42B37, 26D10 31A10, 35J25, 35J30 35K10, 35L10, 35R05 74J15, 76D, 74B05.

Keywords. Sobolev spaces, Capacitary inequality, Hardy inequality, Riesz potential space, Singular integral operator, Fredholm radius, Maximal operator, Boundary value problems for elliptic operators, Laplace equation, Cauchy problem, Lipschitz domains, Jordan domains, Green function, Capacity potential, Polyharmonic potential, Oblique derivative problem, Lame system, Stokes system, Navier-Stokes system, Wiener test, $p$-Laplace operator, Heat operator, Wave operator, Surface waves, Hybrid iterative methods.

\section{Contents}

1. Introduction

2. Basic Notations 4

3. Sobolev Spaces of Integer and Fractional Orders 5

3.1. Isoperimetric Problem for Fractional Perimeter 5

3.2. Strong Capacitary Inequality for Functions with Arbitrary Boundary Data

3.3. Boundary Traces of Functions in a Sobolev Space

3.4. Embedding of a Sobolev Space into the Space of Distributions 7

3.5. An Approximation Problem in the Theory of Sobolev Spaces 7

3.6. Extensions of Sobolev Functions Outside of an Angle 7

3.7. Best Constants in Some Classical Inequalities 8

3.8. Hardy Inequality with Capacitary Distance to $\partial \Omega \quad 8$

The author would like to thank O.O. Ibrogimov, M. Mitrea and P. Kuchment for the help in editing this article. 
3.9. Uncertainty Principle for Divergence-Free Fields 9

3.10. Normalization of an Anisotropic Riesz Potential Space 9

3.11. Two-Weight Hardy Inequality Involving First and Second Derivatives

3.12. Trace Inequality with a Non-Positive Measure 10

3.13. Domains for Which $L^{l, p}(\Omega) \cap L^{\infty}(\Omega)$ is a Banach Algebra 10

4. Integral Operators 11

4.1. A Multi-Dimensional Integral Equation Which can be Reduced to One-Dimensional

4.2. A Singular Integral Operator in $L^{p}\left(\mathbb{R}^{n}\right) \quad 11$

4.3. Fredholm Radius of the Harmonic Double Layer Potential 11

4.4. Potential Theory for Surfaces with Cusps 12

4.5. Regularity of Domains in the Theory of Boundary Integral Equations

4.6. Integral Equations of Potential Theory Under Interaction of Nearly Touching Particles

4.7. Non-Classical Maximal Operator

4.8. Fourier $p$-Capacity and Potentials 13

5. Linear Elliptic Operators 13

5.1. Integral Inequality for the Integrable Laplacian 13

5.2. Hölder Regularity of a Boundary Point with Respect to an Elliptic Operator of Second Order

5.3. Uniqueness in the Cauchy Problem for the Laplace Equation 15

5.4. De Giorgi-Nash Theorem for Equations with Complex-Valued Coefficients

5.5. Fractal Domains Versus Lipschitz Domains

5.6. Dirichlet Problem in a Planar Jordan Domain 16

5.7. Hölder Regularity of Capacitary Potential of $n$-Simensional Cube

5.8. Non-Lipschitz Graph Domains Which are Weakly Lipschitz 17

5.9. Self-Adjointness of the Laplacian in a Weighted $L^{2}$-Space 17

5.10. Peculiarities of Planar $C^{1}$ Domains and Extensions of the Dirichlet Laplacian

5.11. Pointwise Estimates for Polyharmonic Green's Functions 18

5.12. Boundedness of Solutions to the Polyharmonic Equation in Arbitrary Domains

5.13. Polyharmonic Capacitary Potentials 19

5.14. Subadditivity of the Polyharmonic Capacity 20

5.15. Normal Derivative of a Harmonic Function at a Boundary Point

5.16. Essential Spectrum of a Perturbed Polyharmonic Operator

5.17. Singularity of Green's Function for Second Order Elliptic Equations with Lower Order Terms

5.18. Generic Degenerating Oblique Derivative Problem 21

5.19. Matrix Generalization of the Oblique Derivative Problem 23

5.20. Coercive Weighted Estimates for Elliptic Equations in Domains with Inner Cusps 
5.21. Multiplicative Inequality for the Linear Elasticity System in an Arbitrary Domain

5.22. $L^{p}$-Dissipativity of the Lamé Operator

5.23. Generalized Maximum Principle for the Lamé System in a Domain with Non-Lipschitz Graph Conical Vertex

5.24. Boundedness of the Displacement Vector in the Traction Problem in Domains with Non-Lipschitz Graph Conical Vertices

5.25. Comparison of Martin's and Euclidian Topologies 25

6. Nonlinear Elliptic Operators 25

6.1. Nonlinear Singularities at the Vertex of a Cone 25

6.2. Nonlinear Boundary Value Problem with Infinite Dirichlet Data 26

6.3. Singularities of Solutions to the Neumann Problem for a Semilinear Equation

6.4. Positive Solutions of a Non-Linear Dirichlet Problem with Zero Boundary Data

6.5. Positive $p$-Harmonic Functions in a Cone 27

6.6. Phragmen-Lindelöf Principle for the $p$-Laplace equation 27

6.7. Isolated Singularity of Solution to a Nonlinear Elliptic System 28

6.8. Poisson Type Solutions of Riccati's Equation with Dirichlet Data

7. Parabolic, Hyperbolic and General Differential Operators

7.1. Behaviour at Infinity of Solutions to the Cauchy-Dirichlet

Problem for a Semi-Linear Parabolic Equation

7.2. Two Inequalities Related to the Heat Equation

7.3. Trace Space for the Maximal Operator Generated by the Wave Operator

7.4. Characteristic Problem for Nonlinear Hyperbolic Operators 30

7.5. Boundary Traces of Functions in the Domain of a Maximal Differential Operator with $t$-Dependent Coefficients $\quad 30$

7.6. Differential Operators Acting in Pairs of Sobolev Spaces 31

7.7. Existence of a Well-Posed Boundary Value Problem for for General Differential Operators in $L^{p}(\Omega)$

7.8. Existence of a Well-Posed Boundary Value Problem for Unbounded Domains

8. Stationary Stokes and Navier-Stokes Systems

8.1. Hölder Regularity of Solutions to the $n$-Dimensional Dirichlet-

Stokes Problem

8.2. Differentiability of Solutions to the Dirichlet Problem for the

Equations of Hydrodynamics in Convex Domains

8.3. Boundedness of Solutions to the Dirichlet Problem for the Stokes System in Arbitrary 3D Domains

8.4. Resolvent $L^{p}$-Estimates for the Dirichlet-Stokes Operator

8.5. Non-Uniqueness for the Stationary Navier-Stokes System with

Dirichlet Data 
9.1. Well-Posed Neumann-Kelvin Problem for a Surface-Piercing Body

9.2. Solvability of the Two-Dimensional Kelvin-Neumann Problem for a Submerged Cylinder

9.3. Counterexample in the Water-Wave Problem for a Submerged Body

9.4. Sharp Hardy-Leray Inequality for Divergence-Free Fields 35

10. Miscellaneous Topics

10.1. A Modified Riemann's Zeta Function Appearing in a NonLocal Parabolic Cauchy Problem

10.2. Uniqueness Criterion for Analytic Functions with Finite Dirichlet Integral

10.3. Hybrid Iterative Methods for Solving Boundary Value Problems

10.4. Asymptotic Optimization of Multi-Structures 37 References

\section{Introduction}

The title of this article was inspired by the title of Sholem Aleichem's story "Seventy five thousand". Alas, the reader will find only seventy five problems here! I hope each of them can produce a long sequence (a thousand) of others.

The problems below present a sample of questions that I came across upon during many years of research, but have never seen them solved or even clearly formulated. Some of them might turn out being rather simple, while others would present a significant challenge and hopefully attract interest of beginning researchers and experts alike.

I tried to select questions that can be formulated without much preliminary knowledge. For more explanations the reader is directed to the cited literature.

The problems included here address both linear and non-linear PDEs. One can hear sometimes that "all linear problems had been already solved". Hopefully, the following list of problems will show that this opinion is mistaken.

\section{Basic Notations}

Here some notations which are used in the article are collected.

By a domain $\Omega$ we mean an open connected set in $\mathbb{R}^{n}$ with boundary $\partial \Omega$. We say that $\Omega$ is a Lipschitz graph domain if it can be locally explicitly represented in a certain cartesian system by a Lipschitz function. The domain is weakly Lipschitz if it is locally Lipschitz diffeomorphic to a ball. Let $H_{n-1}$ stand for the $(n-1)$-dimensional Hausdorff measure. Let $B_{r}(x)$ denote the open ball with center $x$ and radius $r, B_{r}=B_{r}(O)$, where $O$ is the origin. We shall use the notation $m_{n}$ for $n$-dimensional Lebesgue measure and $v_{n}$ for 
$m_{n}\left(B_{1}\right)$. We use the notation $\nabla_{l} u$ for the distributional gradient of order $l$, i.e. $\nabla_{l} u=\left\{D^{\alpha} u\right\}$, where $\alpha$ is a multi-index $\left(\alpha_{1}, \ldots, \alpha_{n}\right), D^{\alpha}=D_{x_{1}}^{\alpha_{1}} \ldots D_{x_{n}}^{\alpha_{n}}$ and $|\alpha|=\sum_{j} \alpha_{j}=l$. We put $\nabla=\nabla_{1}$.

Let $1 \leq p<\infty, l \in \mathbb{N}$, and let $\Omega$ be a domain in $\mathbb{R}^{n}$. By $L^{l, p}(\Omega)$ we denote the space of distributions whose gradient of order $l$ is in $L^{p}(\Omega)$ supplied with the norm

$$
\left\|\nabla_{l} u\right\|_{L^{p}(\Omega)}+\|u\|_{L^{p}(\omega)},
$$

where $\omega$ is a bounded subdomain of $\Omega$ with $\operatorname{dist}(\partial \omega, \partial \Omega)>0$.

We call the quantities $a$ and $b$ equivalent if there are positive constants $c_{1}$ and $c_{2}$ such that $c_{1} a \leq b \leq c_{2} a$.

By $c, C$ we denote different positive constants.

The Einstein summation convention is always assumed.

\section{Sobolev Spaces of Integer and Fractional Orders}

\subsection{Isoperimetric Problem for Fractional Perimeter}

Let $\Omega$ be a bounded domain with smooth boundary $\partial \Omega$ and $\alpha \in(0,1)$. The following set function was considered in [68]:

$$
P_{\alpha}(\Omega)=\int_{\Omega} \int_{\mathbb{R}^{n} \backslash \Omega} \frac{d x d y}{|x-y|^{n+\alpha}} .
$$

Later, $P_{\alpha}(g)$ was called the "fractional perimeter of $\Omega$ " and has attracted a lot of attention since.

By [68, Section 6], (see also [71, Section 11.10]), the best constant $C$ in the isoperimetric inequality

$$
P_{\alpha}(\Omega) \leq C H_{n-1}(\partial \Omega)^{\frac{n-\alpha}{n-1}}
$$

is the same as the best constant $C$ in the functional inequality

$$
\left(\int_{\mathbb{R}^{n}} \int_{\mathbb{R}^{n}} \frac{|u(x)-u(y)|^{q}}{|x-y|^{n+\alpha}} d x d y\right)^{1 / q} \leq 2^{1 / q} C\|\nabla u\|_{L^{1}\left(\mathbb{R}^{n}\right)}
$$

where $q=n /(n-\alpha)$ and $u \in C_{0}^{\infty}\left(\mathbb{R}^{n}\right)$.

By [68, p. 333],

$$
C \leq \frac{n v_{n}^{(n-1+\alpha) / n}}{\alpha(1-\alpha)}\left(H_{n-1}\left(\partial B_{1}\right)\right)^{\frac{n-\alpha}{n-1}} .
$$

The constant factor on the right, although explicit, is hardly best possible.

Problem 1. Find the best value of the isoperimetric constant $C$ in (3.1). 


\subsection{Strong Capacitary Inequality for Functions with Arbitrary Boundary Data}

Let $F$ be a relatively closed subset of $\Omega$. By the capacity of $F$ generated by the norm in $L^{l, p}(\Omega)$ we mean the set function

$$
\operatorname{cap}\left(F, L^{l, p}(\Omega)\right)=\inf \left\{\|u\|_{L^{l, p}(\Omega)}^{p}: u \in C^{\infty}(\Omega),\left.u\right|_{F} \geq 1\right\} .
$$

Similar capacities are frequently used in potential theory, partial differential equations and theory of function spaces (see $[60,69,70]$ ).

Here is, for example, a simple application of capacity to the integral inequality

$$
\|u\|_{L^{p}(\Omega, \mu)} \leq c\|u\|_{L^{l, p}(\Omega)},
$$

where $p \in(1, \infty), \mu$ is a Borel measure on $\Omega$ and $u \in C^{\infty}(\Omega)$ is an arbitrary function. Take a relatively closed subset $F$ of $\Omega$ and any function $u$ from the above definition of $\operatorname{cap}\left(F, L^{l, p}(\Omega)\right)$. Then (3.2) implies

$$
\mu(F) \leq C \operatorname{cap}\left(F, L^{l, p}(\Omega)\right) .
$$

Thus, this isocapacitary isoperimetric inequality proves to be a necessary condition for inequality (3.3).

It is easy to show that the condition (3.4) is sufficient for (3.3) provided that the so-called strong capacitary inequality

$$
\int_{0}^{\infty} \operatorname{cap}\left(\{x \in \Omega:|u(x)| \geq t\}, L^{l, p}(\Omega)\right) d\left(t^{p}\right) \leq c\|u\|_{L^{l, p}(\Omega)}^{p}
$$

holds for all $u \in C^{\infty}(\Omega)$.

For functions with zero boundary conditions inequalities of type (3.5) first appeared in [61] (see also [69] and [1]). If $l=1,(3.5)$ holds without restrictions on $\Omega$. However, already for $l>1$ the domain should satisfy some regularity conditions as the counterexample constructed in [80] shows (see also [83, Section 2.13]). This gives rise to the following

Problem 2. Find necessary and sufficient conditions, or only non-trivial sufficient conditions, on $\Omega$ guaranteeing the inequality (3.5).

\subsection{Boundary Traces of Functions in a Sobolev Space}

Let $\Omega$ be a bounded weakly Lipschitz domain and let $\varphi: \partial \Omega \rightarrow \mathbb{R}^{1}$.

It is well-known that $\varphi$ is the trace of a function in $L^{1, p}(\Omega)$, if and only if $\varphi \in L^{1}(\partial \Omega)$ for $p=1$ and $\varphi \in L^{1-1 / p, p}(\partial \Omega)$ for $p>1$, where the Besov space $L^{\alpha, p}(\partial \Omega), 0<\alpha<1$, is supplied with the seminorm

$$
\left(\int_{\partial \Omega} \int_{\partial \Omega}|\varphi(x)-\varphi(y)|^{p} \frac{d s_{x} d s_{y}}{|x-y|^{n+\alpha p-2}}\right)^{1 / p} .
$$

This fact fails if $\Omega$ is not weakly Lipschitz. Other characterizations of the trace are given for some classes of non-Lipschitz domains in [83, Chapter 6].

Problem 3. Justify the following conjecture concerning an "extremal length"1 characterization of traces for an arbitrary domain $\Omega$ : The function $\varphi$ is the trace of a function in $L^{1, p}(\Omega)$ if and only if there exists a function $\rho \in C(\Omega) \cap$

\footnotetext{
${ }^{1}$ For the notion "extremal length" see [17].
} 
$L^{p}(\Omega)$ such that for "quasi-every" pair of points $x$ ' and $x$ " on $\partial \Omega$ and for any locally rectifiable arc $\gamma \subset \Omega$ joining $x^{\prime}$ and $x^{\prime \prime}$ there holds the inequality

$$
\left|\varphi\left(x^{\prime}\right)-\varphi\left(x^{\prime \prime}\right)\right| \leq \int_{\gamma} \rho(x)|d x| .
$$

By "quasi-everywhere" one means outside of a set of zero $L^{1, p}$-capacity.

\subsection{Embedding of a Sobolev Space into the Space of Distributions}

Let $\Omega$ be an open set in $\mathbb{R}^{n}$. By $L_{0}^{l, p}(\Omega, \mu)$ we denote the completion of $C_{0}^{\infty}(\Omega)$ in the norm

$$
\left\|\nabla_{l} u\right\|_{L^{p}(\Omega)}+\left(\int_{\Omega}|u|^{p} d \mu\right)^{1 / p}
$$

where $p \in[1, \infty]$ and $\mu$ is a measure.

The question of embedding $L_{0}^{l, p}(\Omega, 0)$ into the distribution space $\left[C_{0}^{\infty}(\Omega)\right]^{\prime}$ is completely solved in [23] for $p=2$ and by [69, Theorem 15.2] for all values of $p$.

Problem 4. Find necessary and sufficient conditions on the measure $\mu$ ensuring the embedding of $L_{0}^{l, p}(\Omega, \mu)$ into $\left[C_{0}^{\infty}(\Omega)\right]^{\prime}$.

\subsection{An Approximation Problem in the Theory of Sobolev Spaces}

Lewis [43] showed that $C^{\infty}(\bar{\Omega})$ is dense in $L^{1, p}(\Omega)$ if $\Omega$ is an arbitrary Jordan domain in the plane, i.e. $\partial \Omega$ is homeomorphic to $\partial B_{1}$. In [44] he asked if $C^{\infty}(\bar{\Omega})$ is dense in $L^{l, p}(\Omega)$ with $l>1$ for every Jordan domain $\Omega \subset \mathbb{R}^{2}$.

It is almost obvious that $L^{\infty}(\Omega) \cap L^{1, p}(\Omega)$ is dense in $L^{1, p}(\Omega)$ for every open set $\Omega \subset \mathbb{R}^{n}$. A direct extension of this property for $L^{l, p}(\Omega)$ with $l>1$ is impossible, as shown in [80]. A planar domain $\Omega$ in the counterexample proposed in [80] is not Jordan, which gives rise to the following

Problem 5. Are bounded functions belonging to $L^{l, p}(\Omega), l>1$, dense in $L^{l, p}(\Omega)$ if $\Omega$ is an arbitrary Jordan domain?

\subsection{Extensions of Sobolev Functions Outside of an Angle}

Let $A_{\alpha}$ denote the angle $\{z \in \mathbb{C}: 0<\arg z<\alpha\}, 0<\alpha<2 \pi$, and let $\mathcal{E}_{\alpha}$ be the extension operator $L^{1,2}\left(A_{\alpha}\right) \rightarrow L^{1,2}\left(\mathbb{R}^{2}\right)$ which has the least norm. Using the Radon transform, one can show that

$$
\left\|\mathcal{E}_{\alpha}\right\|=\max \left\{\left(\frac{\alpha}{2 \pi-\alpha}\right)^{1 / 2},\left(\frac{2 \pi-\alpha}{\alpha}\right)^{1 / 2}\right\} .
$$

Problem 6. Find the least norm of the extension operator $W^{1,2}\left(A_{\alpha}\right) \rightarrow$ $W^{1,2}\left(\mathbb{R}^{2}\right)$, where

$$
\|u\|_{W^{1,2}(\Omega)}=\left(\|\nabla u\|_{L^{2}(\Omega)}^{2}+\|u\|_{L^{2}(\Omega)}^{2}\right)^{1 / 2} .
$$




\subsection{Best Constants in Some Classical Inequalities}

Consider the inequality

$$
\int_{\partial B_{1}}|u(x)|^{2} d \omega \leq C \int_{B_{1}}|\nabla u|^{2} d x
$$

where $B_{1}$ is the open unit ball in $\mathbb{R}^{n}, n>2, d \omega$ is an element of the surface area on the sphere $\partial B_{1}$, and $u$ is an arbitrary $C^{0,1}$ (Lipschitz) function given on $\overline{B_{1}}$ that vanishes on a closed subset $F$ of $\overline{B_{1}}$. This inequality holds if and only if the harmonic (Wiener) capacity $\operatorname{cap}(F)$ is positive, where the Wiener capacity is defined as follows

$$
\operatorname{cap}(F)=\inf \left\{\int_{\mathbb{R}^{n}}|\nabla v|^{2} d x: v \in C^{0,1}\left(\mathbb{R}^{n}\right), v \geq 1 \text { on } F\right\} .
$$

The following assertion containing an explicit value of $C$ in (3.6) was obtained in [51], see also [91].

Let $u \in C^{0,1}\left(\overline{B_{1}}\right), u=0$ on $F$, where $F \subset \overline{B_{1}}$. Then

$$
\int_{\partial B_{1}}|u(x)|^{2} d \omega \leq \frac{c_{n}}{\operatorname{cap}(F)} \int_{B_{1}}|\nabla u|^{2} d x,
$$

where

$$
c_{n}=2(n-1) n v_{n} .
$$

Various inequalities related to (3.7) have interesting applications to the theory of partial differential equations and were studied from different points of view in [71] and elsewhere. However, the question of sharp constants has never been addressed. Therefore, it seems reasonable to formulate the problem.

Problem 7. Find the best value of $c_{n}$ in (3.7).

\subsection{Hardy Inequality with Capacitary Distance to $\partial \Omega$}

Let $\Omega$ be a domain in $\mathbb{R}^{n}, n>2$. As is well-known, the Hardy inequality

$$
\int_{\Omega}\left(\frac{|u(x)|}{d(x)}\right)^{2} d x \leq c \int_{\Omega}|\nabla u|^{2} d x
$$

where $u \in C_{0}^{\infty}(\Omega)$ and $d(x)$ is the distance from $x$ to $\partial \Omega$, does not hold for any domain and conditions for its validity are known (see [69, p. 733-735] for references).

Problem 8. Prove that (3.9) holds for any domain $\Omega$ if $d(x)$ is replaced by the capacitary distance defined by

$$
d_{\alpha}(x)=\inf \left\{r>0: \operatorname{cap}\left(B_{r}(x) \backslash \Omega\right) \geq \alpha \operatorname{cap}\left(B_{r}\right)\right\},
$$

where $\alpha$ is a positive parameter and cap denotes the Wiener capacity defined in the previous subsection. 


\subsection{Uncertainty Principle for Divergence-Free Fields}

The inequality

$$
\left(\int_{\mathbb{R}^{1}} u^{2} d x\right)^{2} \leq 4\left(\int_{\mathbb{R}^{1}} x^{2} u^{2} d x\right)\left(\int_{\mathbb{R}^{1}}\left(u^{\prime}\right)^{2} d x\right)
$$

is the so-called uncertainty principle in quantum mechanics, and the constant 4 is sharp.

Problem 9. What is the best constant $C>0$ in the inequality

$$
\left(\int_{\mathbb{R}^{n}}|\mathbf{u}|^{2} d x\right)^{2} \leq C\left(\int_{\mathbb{R}^{n}}|x|^{2}|\mathbf{u}|^{2} d x\right)\left(\int_{\mathbb{R}^{n}}|\nabla \mathbf{u}|^{2} d x\right),
$$

where $\mathbf{u}=\left(u_{1}, \ldots, u_{n}\right)$ is an arbitrary divergence-free vector field?

Answer the same question concerning the inequality

$$
\left(\int_{\mathbb{R}^{n}}|\mathbf{u}|^{p} d x\right)^{2} \leq C_{p}\left(\int_{\mathbb{R}^{n}}(|x||\mathbf{u}|)^{p /(p-1)} d x\right)^{p-1}\left(\int_{\mathbb{R}^{n}}|\nabla \mathbf{u}|^{p} d x\right),
$$

where $p \in(1, \infty)$ and $\operatorname{div} \mathbf{u}=0$ in $\mathbb{R}^{n}$.

\subsection{Normalization of an Anisotropic Riesz Potential Space}

Let $1<p<\infty$ and let $\mathbf{l}=\left(l_{1}, \ldots, l_{n}\right)$, where $0<l_{i}<1$. By $L^{\mathbf{l}, p}\left(\mathbb{R}^{n}\right)$ we denote the generalization of the classical Riesz potential space normed by

$$
\|\mu(D) u\|_{L^{p}\left(\mathbb{R}^{n}\right)},
$$

where $\mu(D)$ is the pseudodifferential operator with symbol

$$
\mu(\lambda)=\sum_{i=1}^{n}\left|\lambda_{i}\right|^{l_{i}}
$$

It is shown in [48] (see also [24]) that the space $L^{\mathbf{l}, 2}\left(\mathbb{R}^{n}\right)$ can be endowed with the norm $\left\|D^{1,2} u\right\|_{L^{2}\left(\mathbb{R}^{n}\right)}$, where

$$
\left(D^{1,2}\right) u(x)=\left(\int_{\mathbb{R}^{n}}|u(x+z)-u(x)|^{2}\left(\sum_{i=1}^{n}\left|z_{i}\right|^{l_{i}}\right)^{-\delta} d z\right)^{1 / 2}
$$

with

$$
\delta=2+\sum_{i=1}^{n} l_{i}^{-1} .
$$

This norm is useful in applications such as the description of multipliers in $L^{1,2}\left(\mathbb{R}^{n}\right)$ and the theory of Dirichlet forms.

Problem 10. For $p \neq 2$ find an analog of the just mentioned representation of the norm in $L^{\mathbf{l}, p}\left(\mathbb{R}^{n}\right)$ involving the difference $u(x+z)-u(x)$.

Remark. Strichartz [101] supplied the space of isotropic Riesz potentials with the norm $\left\|S_{l} u\right\|_{L^{p}\left(\mathbb{R}^{n}\right)}$, where

$$
\left(S_{l} u\right)(x)=\left(\int_{0}^{\infty}\left[\int_{|z|<y}|u(x+z)-u(x)| d z\right]^{2} \frac{d y}{y^{1+2(n+l)}}\right)^{1 / 2} .
$$




\subsection{Two-Weight Hardy Inequality Involving First and Second Derivatives}

Let $p \geq 1$ and let $\gamma \neq 1-2 / p$. By $\psi$ we denote a function in $C_{0}^{\infty}\left(\mathbb{R}^{2}\right)$. In case $\gamma<1-2 / p$ we require additionally that $\nabla \psi(0)=0$.

Problem 11. Find the best constant in the inequality

$$
\int_{\mathbb{R}^{2}}|\nabla \psi|^{p}|x|^{p(\gamma-1)} d x \leq C(p, \gamma) \int_{\mathbb{R}^{2}}\left(\psi_{x_{1} x_{1}}^{2}+2 \psi_{x_{1} x_{2}}^{2}+\psi_{x_{2} x_{2}}^{2}\right)^{p / 2}|x|^{p \gamma} d x .
$$

It is proved in [10] (see also [69, p. 229]) that when $p=2$,

$$
C(2, \gamma)= \begin{cases}\frac{1+(1-\gamma)^{2}}{\gamma^{2}\left(3+(1-\gamma)^{2}\right)} & \text { for } \gamma \in[-\sqrt{3}-1, \sqrt{3}-1], \\ \left(\gamma^{2}+1\right)^{-1} & \text { otherwise. }\end{cases}
$$

The method used in the proof of this result cannot be applied to the case $p \neq 2$.

\subsection{Trace Inequality with a Non-Positive Measure}

Let $\Omega$ be a bounded smooth domain in $\mathbb{R}^{n}$ and let $\sigma$ be a real measure, not necessarily positive, even a distribution, supported on $\partial \Omega$.

Problem 12. Let $n>p>1$ and let $u$ be any function in $C^{\infty}(\bar{\Omega})$. Find necessary and sufficient condition on $\sigma$ for the validity of the inequality

$$
\inf _{c \in \mathbb{R}^{1}} \int_{\partial \Omega}|u-c|^{p} d \sigma \leq C \int_{\Omega}|\nabla u(x)|^{p} d x .
$$

A similar problem for the inequality

$$
\int_{\Omega}|u|^{p} d \sigma \leq C \int_{\Omega}|\nabla u(x)|^{p} d x
$$

with $\sigma$ supported on $\Omega$ was solved in [25] for $p=2$ and in [26] for an arbitrary $p>1$.

\subsection{Domains for Which $L^{l, p}(\Omega) \cap L^{\infty}(\Omega)$ is a Banach Algebra}

Let $\Omega$ be a domain in $\mathbb{R}^{n}$. We may ask whether the space $L^{l, p}(\Omega) \cap L^{\infty}(\Omega)$ is an algebra with respect to pointwise multiplication. Clearly, this is the case for $l=1$. Since Stein's extension operator from a Lipschitz graph domain $\Omega$ is continuous as an operator

$$
L^{l, p}(\Omega) \cap L^{\infty}(\Omega) \rightarrow L^{l, p}\left(\mathbb{R}^{n}\right) \cap L^{\infty}\left(\mathbb{R}^{n}\right),
$$

the above question has the affirmative answer for the union of a finite number of Lipschitz graph domains. For example, $\Omega$ can be a bounded domain having the cone property. However, by a counterexample proposed in [80] (see also $[69$, p. $120-121])$, it turns out that the space $L^{l, p}(\Omega) \cap L^{\infty}(\Omega)$ is generally not an algebra.

Problem 13. Find a necessary and sufficient condition on $\Omega \subset \mathbb{R}^{n}$ ensuring that $L^{l, p}(\Omega) \cap L^{\infty}(\Omega)$ is a Banach algebra. 
Remark. One can easily check that $W^{2, p}(\Omega) \cap L^{\infty}(\Omega)$ is an algebra with respect to the pointwise multiplication if and only if $W^{2, p}(\Omega) \cap L^{\infty}(\Omega)$ is imbedded into $W^{1,2 p}(\Omega)$.

\section{Integral Operators}

\subsection{A Multi-Dimensional Integral Equation Which can be Reduced to One- Dimensional}

Let $\varphi$ and $\psi$ be functions defined on a multi-dimensional domain $\Omega$.

Problem 14. Develop a theory of solvability of the integral equation

$$
\lambda u(x)-\int_{\Omega} \frac{u(y)}{\varphi(x)-\psi(y)} d y=f(x),
$$

where $f$ is a given function and $\lambda$ is a complex parameter.

Note that (4.1) can be reduced to a certain one-dimensional integral equation by using the so-called coarea formula, see [69].

\subsection{A Singular Integral Operator in $L^{p}\left(\mathbb{R}^{n}\right)$}

Consider the singular integral operator $A$ with the symbol

$$
\partial B_{1} \ni \omega \rightarrow \exp (i \lambda \phi(\omega)),
$$

where $\phi$ is a smooth real-valued function on $\partial B_{1}$, and $\lambda$ is a large real parameter.

Problem 15. Prove or disprove the estimate

$$
\|A\|_{L^{p}\left(\mathbb{R}^{n}\right) \rightarrow L^{p}\left(\mathbb{R}^{n}\right)} \leq c|\lambda|^{(n-1)\left|\frac{1}{2}-\frac{1}{p}\right|}
$$

where $1<p<\infty$, c depends on $n, p$ and the function $\phi$.

\subsection{Fredholm Radius of the Harmonic Double Layer Potential}

Considering the logarithmic harmonic potentials of the single and double layer on curves of "bounded rotation", J. Radon has introduced the notion of the essential norm and the Fredholm radius of a bounded operator in 1919 .

The essential norm $\|L\|$ of a linear bounded operator $L$ acting on a Banach space $\mathcal{B}$ is defined as

$$
\|L\|=\inf _{\{\mathcal{K}\}}\|L-\mathcal{K}\|_{\mathcal{B} \rightarrow \mathcal{B}}
$$

where $\{\mathcal{K}\}$ is the set of all linear compact operators on $\mathcal{B}$.

The Fredholm radius $\mathcal{R}(L)$ of the operator $L$ is the radius of the largest circle on the complex $\lambda$-plane centered at $\lambda=0$ inside which $I+\lambda L$ is a Fredholm operator.

The essential norm $\|L\|$ is related to $\mathcal{R}(L)$ by the inequality

$$
\mathcal{R}(L) \geq\|L\|^{-1} \text {. }
$$


It was shown by Gohberg and Marcus [20] that the Fredholm radius of the operator $L$, acting on a Banach space $\mathcal{B}$ equipped with the norm $\|\cdot\|_{0}$, satisfies

$$
\mathcal{R}(L)=\sup \left\{\|L\|^{-1}:\|\cdot\| \in \mathcal{N}\left(\|\cdot\|_{0}\right)\right\},
$$

where $\|L\|$ is calculated with respect to a norm $\|\cdot\|$ in the set $\mathcal{N}\left(\|\cdot\|_{0}\right)$ of all norms in $\mathcal{B}$, equivalent to $\|\cdot\|_{0}$.

Král [35] and Burago and Maz'ya [5] showed that for a $n$-dimensional bounded domain $\mathcal{D}$ with finite variation of the solid angle $\omega_{\mathcal{D}}(p, E)(p \in$ $\left.\partial \mathcal{D}, E \subset \mathbb{R}^{n}\right)$ the essential norm of the harmonic double layer potential in the Banach space $C(\partial \mathcal{D})$ is

$$
\frac{2}{n v_{n}} \lim _{\delta \rightarrow 0} \sup _{p \in \partial \mathcal{D}} \operatorname{var} \omega_{\mathcal{D}}\left(p, B_{\delta}(p)\right) .
$$

References to other results in the same area can be found in [36, Chapter 7].

Problem 16. Find a geometrical representation for the Fredholm radius of the harmonic double layer potential as an operator in $C(\partial \mathcal{D})$.

\subsection{Potential Theory for Surfaces with Cusps}

A detailed theory of boundary integral equations for harmonic and elastic singe and double layer potentials on curves with exterior and interior cusps was constructed in [93].

Problem 17. Develop a theory of multidimensional boundary integral equations for domains with inner and outer cusps.

First results concerning the multidimensional case can be found in [84-86].

\subsection{Regularity of Domains in the Theory of Boundary Integral Equations}

In [90] (see also [88, Chapter 15]) a regularity theory of classical boundary integral equations involving harmonic, elastic and hydrodynamic potentials on nonsmooth surfaces is presented. A goal of this theory is to establish solvability in fractional Besov spaces.

Problem 18. Obtain results on the solvability of just mentioned equations in Sobolev spaces $L^{l, p}(\partial \Omega)$ with integer $l$ under minimal requirements on $\partial \Omega$.

\subsection{Integral Equations of Potential Theory Under Interaction of Nearly Touching Particles}

The following difficulty arises when using the boundary element method to study hydrodynamic interactions among particles in suspensions and other similar phenomena when the particles are nearly touching. Integration of kernels of integral operators on elements near the closest contact points destroys the accuracy of the numerical procedure.

Problem 19. Find asymptotic representation for densities of harmonic, elastic, and hydrodynamic potentials satisfying the boundary integral equations by using the distance between particles as a small parameter. 


\subsection{Non-Classical Maximal Operator}

Let the maximal operator $M^{\diamond}$ be defined by

$$
\left(M^{\diamond} f\right)(x)=\sup _{r>0}\left|f_{B_{r}(x)} \frac{y-x}{|y-x|} f(y) d y\right|, \quad x \in \mathbb{R}^{n},
$$

where $f$ is locally integrable in $\mathbb{R}^{n}$ and the barred integral stands for the mean value. It is obviously dominated by the Hardy-Littlewood maximal operator $M$. A simple property of $M^{\diamond}$ is the sharp pointwise inequality for functions of one variable

$$
\left|u^{\prime}(x)\right|^{2} \leq \frac{8}{3}\left(M^{\diamond} u\right)(x)\left(M^{\diamond} u^{\prime \prime}\right)(x),
$$

see $[71$, formula $(12.0 .5)]$.

Clearly, $M^{\diamond}$ is dominated by the sharp maximal operator $M^{\sharp}$ defined by

$$
\left(M^{\sharp} f\right)(x)=\sup _{r>0} f_{B_{r}(x)}\left|f(y)-f_{B_{r}(x)}\right| d y,
$$

where $f_{B_{r}(x)}$ stands for the mean value of $f$ in the ball $B_{r}(x)$.

Problem 20. Does there exist a Banach space B such that one of the operators $M^{\sharp}$ or $M^{\diamond}$ is bounded in $B$ whereas the other operator is not bounded?

\subsection{Fourier $p$-Capacity and Potentials}

It is difficult to overestimate the role of various capacities of sets in analysis and partial differential equations.

Let us introduce the set function

$$
\inf \left\{\|\mathcal{F} u\|_{L^{p}\left(\mathbb{R}^{n}\right)}: u \in C_{0}^{\infty}\left(\mathbb{R}^{n}\right), u \geq 1 \text { on } K\right\}
$$

where $p \in[1, \infty], \mathcal{F}$ is the Fourier transform and $K$ is a compact set in $\mathbb{R}^{n}$. It is natural to call (4.2) the Fourier capacity of $K$. If $p=2$, this capacity is equal to $\left(m_{n} K\right)^{1 / 2}$.

Problem 21. Find upper and lower geometrical estimates of the Fourier pcapacity. Develop a theory of Fourier p-potentials

$$
\mathcal{F}^{-1}\left(|\mathcal{F} \mu|^{1 /(p-1)} \mathcal{F} \mu\right),
$$

where $\mu$ is a measure, in spirit of [73-75].

\section{Linear Elliptic Operators}

\subsection{Integral Inequality for the Integrable Laplacian}

It is shown in [89] that the inequality

$$
\left|\int_{\mathbb{R}^{2}} \sum_{i, j=1}^{2} a_{i, j} u_{x_{i}} \overline{u_{x_{j}}} d x\right| \leq C\left(\int_{\mathbb{R}^{2}}|\Delta u| d x\right)^{2},
$$

where $a_{i, j}$ are constants and $u$ is an arbitrary complex-valued function in $C_{0}^{\infty}\left(\mathbb{R}^{2}\right)$, holds if and only if $a_{1,1}+a_{2,2}=0$. 
Other results of a similar nature are obtained in [70], where in particular it is proved that the inequality

$$
\left|\int_{\mathbb{R}^{n}} \sum_{j=1}^{n} \alpha_{j}\right| \frac{\partial u}{\partial x_{j}}\left|\frac{d x}{|x|}\right| \leq C \int_{\mathbb{R}^{n}}|\Delta u| d x,
$$

where $\alpha_{j}$ are real constants, holds for all real-valued functions $u \in C_{0}^{\infty}\left(\mathbb{R}^{n}\right)$ if and only if

$$
\sum_{j=1}^{n} \alpha_{j}=0
$$

Problem 22. I conjecture that the inequality

$$
\left|\int_{\mathbb{R}^{n}} \Phi(\nabla u) d x\right|^{\frac{n-1}{n}} \leq \int_{\mathbb{R}^{n}}|\Delta u| d x,
$$

where $n>1, \Phi$ is a smooth function outside the origin and positive homogeneous of degree $n /(n-1)$, holds for all $u \in C_{0}^{\infty}\left(\mathbb{R}^{n}\right)$ if and only if

$$
\int_{\partial B_{1}} \Phi(x) d \omega_{x}=0 .
$$

\subsection{Hölder Regularity of a Boundary Point with Respect to an Elliptic Op- erator of Second Order}

Let $\Omega$ be a bounded domain in $\mathbb{R}^{n}, n \geq 2$. We fix a non-isolated point $O \in \partial \Omega$ as the origin. Let us say that a function $u$ defined on $\Omega$ is $\alpha$-Hölder continuous at $O$ with $\alpha>0$ if it has a limit $u(O)$ as $x \rightarrow O$ and there exists $\alpha$ such that

$$
|u(x)-u(O)| \leq C|x|^{\alpha}
$$

for all $x \in \Omega$. Similarly, a function $\varphi$ given on $\partial \Omega$ is called $\alpha$-Hölder continuous at $O$ if there is a limit $\varphi(O)$ of $\varphi(x)$ as $x \rightarrow O, x \in \partial \Omega$, and

$$
|\varphi(x)-\varphi(O)| \leq C|x|^{\alpha}
$$

By $u_{\varphi}$ we mean a bounded solution to the Dirichlet problem

$$
L u=0 \quad \text { in } \Omega, \quad u=\varphi \text { on } \partial \Omega,
$$

where $\varphi$ is a bounded Borel function on $\partial \Omega$ and

$$
(L u)(x)=\operatorname{div}(\mathcal{A}(x) \nabla u(x))
$$

is a uniformly elliptic operator with a measurable symmetric coefficient matrix $\mathcal{A}$. Basic facts concerning solvability of this problem can be found in [45].

We introduce the $L$-harmonic measure $\mathcal{H}_{L}(x, \mathcal{B})$, where $x \in \Omega$ and $\mathcal{B}$ is a Borel subset of $\partial \Omega$ (see e.g. [45, Definition 1.2.6]).

Definition. The point $O$ is called

(i) $\alpha$-Hölder regular with respect to $L$ if the $\alpha$-Hölder continuity of $\varphi$ at $O$ implies the $\alpha$-Hölder continuity of $u_{\varphi}$ at $O$,

(ii) Hölder regular with respect to $L$ if there exists $\alpha>0$ such that $O$ is $\alpha$-Hölder regular. 
A necessary and sufficient condition for the Hölder regularity of $O$ is contained in the following assertion proved in [72].

The point $O \in \partial \Omega$ is Hölder regular with respect to $L$ if and only if, for some positive constants $\lambda$ and $C$,

$$
\mathcal{H}_{L}\left(x, \partial \Omega \backslash B_{r}\right) \leq c\left(\frac{|x|}{r}\right)^{\lambda}
$$

for all $r>0$ and $x \in \Omega \cap B_{r}$.

Let $n>2$ and let cap denote the Wiener capacity defined in Sect. 3.7. The following condition, sufficient for the Hölder regularity of $O$ and independent of $L$, was found in $[52,54,55]$ :

$$
\liminf _{r \rightarrow 0} \frac{1}{|\log r|} \int_{r}^{1} \operatorname{cap}\left(\Omega \backslash B_{\rho}\right) \frac{d \rho}{\rho^{n-1}}>0 .
$$

Under additional restrictions on the domain, this condition proves to be necessary for the Hölder regularity, see [54,104].

Clearly, (5.3) fails or works simultaneously for all operators $L$. However, it is not necessary for the Hölder regularity in general, see the counterexample in [66, p. 509-510]. Although (5.2) formally depends on $L$, it is natural to formulate the

Problem 23. Prove or disprove that the Hölder regularity of a point is independent of the operator $L$.

\subsection{Uniqueness in the Cauchy Problem for the Laplace Equation}

Let $\mathbb{R}_{+}^{n+1}=\left\{(p, t): p \in \mathbb{R}^{n}, t>0\right\}$. Suppose a function $u \in C^{1}\left(\overline{\mathbb{R}_{+}^{n+1}} \cup \mathbb{R}^{n}\right)$ is harmonic in $\mathbb{R}_{+}^{n+1}$ and its Dirichlet data has zero of infinite order at the origin. Moreover, let the normal derivative satisfy

$$
\left|\frac{\partial u}{\partial t}(p)\right| \leq h(|p|), \quad p \in \mathbb{R}^{n}
$$

where $h$ is a function defined on $[0, \infty)$ positive on $(0, \infty)$ and such that $h(r) \downarrow 0$ as $r \downarrow 0$. One can ask for which $h$ it is true that (5.4) implies $u=0$.

The following answer to this question was obtained in [76].

Suppose $h$ satisfies certain regularity conditions (not to be specified here), then (5.4) implies $u=0$ if and only if

$$
\int_{0}^{1} \log h(r) d r=-\infty .
$$

By Kelvin's transform, the criterion (5.5) can be stated for harmonic functions in a ball. However, the method of proof given in [76] does not provide an answer to the following question.

Problem 24. Is it possible to replace $\mathbb{R}_{+}^{n+1}$ in the above exposition by a smooth domain? Also, can one replace the Laplace operator by an arbitrary second order elliptic operator with smooth coefficients? 
In this case, it is only known that the uniqueness follows from the estimate

$$
|\nabla u(x)| \leq \exp \left(-1 /|x|^{c}\right)
$$

with a certain positive $c$ (see [39]) and with $c=2+\varepsilon, \varepsilon>0$, (see [41]).

\subsection{De Giorgi-Nash Theorem for Equations with Complex-Valued Coeffi- cients}

Let $\Omega$ be a domain in $\mathbb{R}^{n}$. By the classical De Giorgi-Nash theorem, an arbitrary weak solution of the uniformly elliptic equation

$$
\frac{\partial}{\partial x_{i}}\left(a_{i j}(x) \frac{\partial u}{\partial x_{j}}\right)=0, \quad 1 \leq i, j \leq n
$$

with measurable bounded real-valued coefficients is locally Hölder continuous. If the coefficients $a_{i j}$ are complex-valued and

$$
\operatorname{Re}\left(a_{i j}(x) \xi_{i} \overline{\xi_{j}}\right) \geq C|\xi|^{2}
$$

for all $x \in \Omega$ and $\xi \in \mathbb{R}^{n}$, this assertion is not true for dimensions $n>4$. See [95, p. 391-393] for examples of equations whose weak solutions are even unbounded near an interior point of a domain.

Problem 25. Prove or disprove that De Giorgi-Nash property holds for dimensions $n=3$ and $n=4$.

The Hölder continuity of $u$ in the case $n=2$ follows from [94].

\subsection{Fractal Domains Versus Lipschitz Domains}

During the last fifty years considerable progress has been made in the study of elliptic boundary value problems on Lipschitz graph domains (see [27] for a survey of this development). In particular, classes of solvability and estimates of solutions received considerable attention in this area.

A Lipschitz graph domain is locally given by

$$
\left\{x=\left(x^{\prime}, x_{n}\right) \in \mathbb{R}^{n}, \quad x_{n}>\varphi\left(x^{\prime}\right)\right\},
$$

where $\varphi$ satisfies the Lipschitz condition. Replacing here Lipschitz condition by Hölder condition, we arrive at the definition of a Hölder graph domain.

With the existing rich theory of boundary value problems in Lipschitz graph domains, the absence of similar results in Hölder graph domains seems ubiquitous.

Problem 26. Address this issue.

\subsection{Dirichlet Problem in a Planar Jordan Domain}

Let $u$ be a variational solution of the Dirichlet problem with zero boundary data for the equation

$$
\mathcal{L}_{2 m}\left(D_{x}\right) u=f
$$

in $\Omega$, where $\Omega$ is an arbitrary bounded Jordan domain in $\mathbb{R}^{2}$ (see Sect. 3.5), $f \in C_{0}^{\infty}(\Omega)$ and $\mathcal{L}_{2 m}$ is a strongly elliptic operator with real constant coefficients. 
Problem 27. Prove that the solution satisfies

$$
\int_{\Omega}\left|\nabla_{m} u\right|^{4-\varepsilon} d x<\infty
$$

for every small $\varepsilon>0$.

This problem is unsolved even for the case of the Laplace operator which is related to Brennan's conjecture [7].

Inequality (5.7) holds for an arbitrary piecewise smooth domain without cusps, see [33, Chapter 9] and [87, Chapter 4].

\subsection{Hölder Regularity of Capacitary Potential of $\boldsymbol{n}$-Simensional Cube}

It can be easily proved by a barrier argument that the harmonic capacitary potential of the cube $\left\{x: 0 \leq x_{i} \leq 1, i=1, \ldots, n\right\}$ belongs to a certain Hölder class $C^{\alpha(n)}, 0<\alpha(n)<1$, which gives rise to the following

Problem 28. Describe the asymptotic behaviour of $\alpha(n)$ as $n \rightarrow \infty$.

\subsection{Non-Lipschitz Graph Domains Which are Weakly Lipschitz}

Recall that open sets, locally Lipschitz diffeomorphic to a half-space, are called weak Lipschitz domains. One can easily construct cones with boundary smooth outside the vertex and polyhedra which are not Lipschitz graph domains (see [33, p. 4]).

Problem 29. Do the known results on elliptic equations in Lipschitz graph domains (see [27] and references therein) extend to arbitrary weakly Lipschitz domains?

It seems, there are no counterexamples to this question and some results concerning non-Lipschitz graph cones suggest the affirmative answer to it, see $[33,87]$.

\subsection{Self-Adjointness of the Laplacian in a Weighted $L^{2}$-Space}

Let $\rho \in L_{\text {loc }}^{1}\left(\mathbb{R}^{n}\right), \rho>0$, and let

$$
L^{2}(\rho)=\left\{u: \int_{\mathbb{R}^{n}}|u(x)|^{2} \rho(x) d x<\infty\right\} .
$$

In 1990 Eidus [14] proved that for $n \geq 3$ a necessary condition for the operator $\rho^{-1} \Delta$ in $L^{2}(\rho)$ to be self-adjoint is

$$
\int_{\mathbb{R}^{n}} \rho(x) d x=\infty .
$$

One can replace $(5.8)$ by

$$
\int_{\mathbb{R}^{n} \backslash F} \rho(x) d x=\infty
$$

for all closed sets $F$ with finite harmonic capacity (see [71, Section 17.1]).

Problem 30. Show that (5.9) is not sufficient and find a sharp sufficient condition for the self-adjointness of $\rho^{-1} \Delta$ in $L^{2}(\rho)$. 


\subsection{Peculiarities of Planar $C^{1}$ Domains and Extensions of the Dirichlet Laplacian}

By $L_{0}^{1,2}(\Omega)$ we denote the completion of $C_{0}^{\infty}(\Omega)$ in the norm of $L^{1,2}(\Omega)$. By $\bar{\Delta}$ we denote the closure of the Laplacian $\Delta: L^{2,2}(\Omega) \cap L_{0}^{1,2}(\Omega) \rightarrow L^{2}(\Omega)$. Let $\tilde{\Delta}$ be the Friedrichs extension of $\Delta$ and let $\Delta^{*}$ denote the adjoint of $\Delta$. It is well known that $\Delta=\bar{\Delta}=\tilde{\Delta}=\Delta^{*}$, if $\Omega$ is sufficiently smooth. This is not true in general if $\Omega$ is not in the class $C^{2}$.

The following result was obtained in $[56,63]$.

Let $\omega(r)$ be the angle measure of $\{x \in \Omega:|x|=r\}$, where $O \in \partial \Omega$ and $\Omega \subset \mathbb{R}^{2}$. If $\partial \Omega \backslash\{O\}$ is smooth and

$$
\int_{0}^{1} \exp \left(\frac{2}{\pi} \int_{\rho}^{1} \frac{\pi-\omega(r)}{r} d r\right) \frac{d \rho}{\rho}=\infty,
$$

then $\bar{\Delta}=\tilde{\Delta}$. This condition is also necessary under some additional restrictions.

Here are two surprising consequences of the above statement.

(i) There exists a planar $C^{1}$ domain $\Omega$ such that the closure of $\Delta$ is selfadjoint, but the estimate

$$
\|u\|_{L^{2,2}(\Omega)} \leq C\|\Delta u\|_{L^{2}(\Omega)}
$$

does not hold for all $u$ in the domain of $\tilde{\Delta}$, i.e. $\bar{\Delta}=\tilde{\Delta}$ but $\Delta \neq \bar{\Delta}$.

(ii) The closure of the operator $(1+\varepsilon) \partial_{x_{1}}^{2}+\partial_{x_{2}}^{2}, \varepsilon>0$, can be non-selfadjoint whilst for the same $\Omega$, the generalized solution $u \in L_{0}^{1,2}(\Omega)$ of the equation $\Delta u=f$ satisfies (5.10) and, consequently, $\Delta=\bar{\Delta}=\tilde{\Delta}$.

Example. Let

$$
\Omega=\left\{x \in B_{1}: x_{2}>\frac{-C\left|x_{1}\right|}{|\log | x_{1}||}\right\} \subset \mathbb{R}^{2},
$$

where $C$ is a positive constant. Then

$$
\begin{array}{lll}
\Delta=\bar{\Delta}=\tilde{\Delta} & \text { if and only if } & C<\pi / 4, \\
\Delta \neq \bar{\Delta}, \bar{\Delta}=\tilde{\Delta} & \text { if and only if } & C=\pi / 4, \\
\Delta \neq \bar{\Delta}, \text { ind } \bar{\Delta}=1 & \text { if and only if } & C>\pi / 4 .
\end{array}
$$

These isolated phenomena were studied only for the particular case $n=2$ and the Laplace-Dirichlet operator

Problem 31. Obtain similar results for various extensions of elliptic differential operators under minimal restrictions on $\Omega$ and the coefficients.

Concerning the index of elliptic boundary value problems in domains with angle and cone vertices see [15,82].

\subsection{Pointwise Estimates for Polyharmonic Green's Functions}

Let $\Omega$ be an arbitrary domain in $\mathbb{R}^{n}$. Green's function of $\Delta^{m}$ is a solution of

$$
\Delta_{x}^{m} G_{m}(x, y)=\delta(x-y), \quad x, y \in \Omega
$$

subject to zero Dirichlet conditions for $x \in \partial \Omega$. Here $\delta$ is the Dirac function. 
Problem 32. Let $n>2 m$. Prove or disprove that

$$
\left|G_{m}(x, y)\right| \leq \frac{c(m, n)}{|x-y|^{n-2 m}}, \quad x, y \in \Omega, x \neq y,
$$

where $c(m, n)$ does not depend on $\Omega$.

This estimate is known for the dimensions $n=5,6,7$ if $m=2$ and $n=2 m+1,2 m+2$ if $m>2$, see [67].

\subsection{Boundedness of Solutions to the Polyharmonic Equation in Arbitrary Domains}

Let $\Omega$ be an arbitrary bounded domain in $\mathbb{R}^{n}$ and suppose $n>2 m+2$ if $m>2$ and $n \geq 8$ if $m=2$.

Problem 33. Prove or disprove that the variational solution of the equation

$$
\Delta^{m} u=f, \quad f \in C_{0}^{\infty}(\Omega),
$$

subject to zero Dirichlet boundary conditions is in $L^{\infty}(\Omega)$.

This is true for the dimensions listed at the end of the previous subsection (see [67]) and fails for the operator

$$
\Delta_{x}^{2}+10 \frac{\partial^{4}}{\partial x_{8}^{4}}
$$

with $x=\left(x_{1}, \ldots, x_{8}\right)$, see [78].

\subsection{Polyharmonic Capacitary Potentials}

Let $m=1,2, \ldots$. The $m$-harmonic capacity of a compact set $F$ in $\mathbb{R}^{n}$ is defined by

$$
\begin{aligned}
\operatorname{cap}_{m}(F) & =\inf \left\{\sum_{|\alpha|=m} \frac{m !}{\alpha !}\left\|\partial^{\alpha} u\right\|_{L^{2}\left(\mathbb{R}^{n}\right)}^{2}: u \in C_{0}^{\infty}(\Omega),\right. \\
u & =1 \text { in a neigborhood of } F\}
\end{aligned}
$$

was introduced in [50] (see also [53]) and proved to be useful in the theory of higher order elliptic equations. It is well-known that $\operatorname{cap}_{m}(F)=0$ for all compact sets $F$ if $2 m \geq n$.

Let $n>2 m$. One of the definitions of the potential-theoretic Riesz capacity of order $2 m$ is as follows:

$$
R_{2 m}(F)=\inf \left\{\sum_{|\alpha|=m} \frac{m !}{\alpha !}\left\|\partial^{\alpha} u\right\|_{L^{2}\left(\mathbb{R}^{n}\right)}^{2}: u \in C_{0}^{\infty}\left(\mathbb{R}^{n}\right), u \geq 1 \text { on } F\right\} .
$$

The capacities $\operatorname{cap}_{m}(F)$ and $R_{2 m}(F)$ are equivalent, that is their ratio is bounded and separated from zero by positive constants depending only on $n$ and $m$ (see [57,62] and [71, Section 13.3]).

The minimizer in $R_{2 m}$ is a Riesz potential of a measure whereas that in $\operatorname{cap}_{m}$ is a Riesz potential of a distribution. The last minimizer will be denoted by $U_{F}$. By [67], for the dimensions $n=5,6,7$ if $m=2$ and $n=2 m+1,2 m+2$ if $m>2$, the inequalities 


$$
0<U_{F}(x)<2
$$

hold for all $x \in \mathbb{R}^{n} \backslash F$. In general, the constant 2 cannot be replaced by 1 .

Problem 34. Is it possible to extend (5.13) for dimensions $n \geq 8$ if $m=2$, and $n \geq 2 m+3$ for $m>2$ ? Another question: is the constant 2 in (5.13) best possible?

\subsection{Subadditivity of the Polyharmonic Capacity}

Let $\operatorname{cap}_{m}(F)$ and $R_{2 m}(F)$ be the capacities defined by (5.11) and (5.12). By the classical potential theory, the Riesz capacity $R_{2 m}$ is upper subadditive. However, the following problem is open.

Problem 35. Prove or disprove that the m-harmonic capacity $\mathrm{cap}_{m}$ is upper subadditive.

\subsection{Normal Derivative of a Harmonic Function at a Boundary Point}

Let a $\delta$-neighborhood of the origin $O \in \partial \Omega$ be given by the inequality $x_{n}>$ $\varphi\left(x^{\prime}\right)$, where $\varphi$ is a Lipschitz function of the variable $x^{\prime} \in \mathbb{R}^{n-1}, \varphi(0)=0$. We assume that the function

$$
\omega(r)=\sup _{\left|x^{\prime}\right|<r}\left|\nabla \varphi\left(x^{\prime}\right)\right|
$$

satisfies

$$
\int_{0}^{\delta} \frac{\omega^{2}(r)}{r} d r<\infty .
$$

Consider also the condition

$$
\int_{|\xi|<\delta} \varphi(\xi) \frac{d \xi}{|\xi|^{n}}<\infty,
$$

where the integral is improper.

The following property of harmonic functions appeared in mathematical literature at the end of the XIX century: if a harmonic function takes its minimum value at $O$, then its inner normal derivative at $O$ is strictly positive.

Problem 36. Prove that under the a priori requirement (5.14), the condition (5.15) is necessary and sufficient for the validity of the just formulated property of harmonic functions.

Note that (5.15) is much weaker than the Dini condition

$$
\int_{0}^{\delta} \frac{\omega(r)}{r} d r<\infty
$$

which previously appeared in the same context (see [2], where other references can be found).

Remark. One possible approach is to use the asymptotic formula for harmonic functions near the Lipschitz boundary established in [30]. 


\subsection{Essential Spectrum of a Perturbed Polyharmonic Operator}

Consider the selfadjoint operator $L$ in $L^{2}\left(\mathbb{R}^{n}\right)$ generated by the differential expression

$$
(-\Delta)^{m}+q(x)
$$

where $q$ is a locally integrable nonnegative function in $\mathbb{R}^{n}$.

Problem 37. Let $m>1$ and $2 m \leq n$. Is it true that either the essential spectrum of $L$ coincides with the half-axis $\lambda \geq 0$, or the point $\lambda=0$ does not belong to the spectrum of $L$ ?

This "spectral alternative" was established by Putnam [98] for $n=1$, $m=1$, by Glazman [19] for $n \geq 1, m=1$, and by Yafaev [107] for $2 m>n$.

\subsection{Singularity of Green's Function for Second Order Elliptic Equations with Lower Order Terms}

It is well-known that Green's function $G(x, y)$ of the uniformly elliptic operator

$$
L u=-\frac{\partial}{\partial x_{i}}\left(a_{i j}(x) \frac{\partial u}{\partial x_{j}}\right), \quad n>2,
$$

with measurable bounded coefficients is equivalent to $|x-y|^{2-n}$ near the point $y$ (see $[45,99])$.

Problem 38. Find necessary and sufficient conditions for the coefficients $b_{1}, \ldots, b_{n}$ and $c$ preserving the equivalence of $|x-y|^{2-n}$ and Green's function of the operator

$$
u \rightarrow L u+b_{i}(x) \frac{\partial u}{\partial x_{i}}+c(x) u .
$$

\subsection{Generic Degenerating Oblique Derivative Problem}

Oblique derivative problem consists in determining solutions of the second order elliptic equation in a domain $\Omega \subset \mathbb{R}^{n}$ subject to the boundary condition

$$
\frac{\partial u}{\partial l}=f \quad \text { on } \partial \Omega
$$

where $l$ denotes a field of unit vectors on $\partial \Omega$. The corresponding boundary pseudodifferential operator is elliptic if and only if $l$ is nowhere tangent to $\partial \Omega$. In the non-elliptic case the oblique derivative problem is called degenerate.

Around 1970 Arnold [3] stressed the importance of the so-called generic case of degeneration, where the vector field $l$ is tangent to $\partial \Omega$ on a submanifold of codimention 1 and is not transversal to this submanifold (see also his well-known book [4, Section 29]).

In [4, Section 29] Arnold writes:

"One of the simplest problems of such a calculation of infinite codimensions corresponding to kernels and cokernels consisting of functions on manifolds of different dimensions is the oblique derivative problem. If we consider this problem on the sphere bounding an $n$-dimensional ball, a vector 
field tangent to the $n$-dimensional ambient space is given. A function harmonic in the ball is to be determined whose derivative in the direction of the field is equal to a given boundary function.

We consider, for example, the case $n=3$. In this case, a generic field is tangent to the sphere on some smooth curve. There are singular points on this curve where the field is tangent to the curve itself. The structure of the field in the neighborhood of each of these singular points is standard: it can be proved that for any $n$ of a generic field in the neighborhood of every point of the boundary, the field is given, in an appropriate coordinate system, by a formula of the form

$$
x_{2} \partial_{1}+x_{3} \partial_{2}+\cdots+x_{k} \partial_{k-1}+\partial_{k}, \quad k \leq n,
$$

where $\partial_{j}=\partial / \partial x_{j}$ and $x_{1}=0$ on the boundary (cf. S.M. Višik, On the oblique derivative problem, Vestnik MGU, Ser. Mathem. 1 (1972), 21-28).

The oblique derivative problem apparently has to be stated according to the following scheme. The manifold of tangency of the field with the boundary, the manifold of the tangency of the field with the tangency manifolds, etc. divide the boundary into parts of various dimensions. On some of these parts of the boundary, conditions have to be given: on some other parts, the boundary function itself has to satisfy certain conditions for the existence of the classical solution of the problem."

In 1972 [59] I published a result related to the generic degeneration, still the only known one. I assumed that there are smooth manifolds

$$
\Gamma_{0} \supset \Gamma_{1} \ldots \supset \Gamma_{s}
$$

of dimensions $n-2, n-3, \ldots, n-2-s$ such that $l$ is tangent to $\Gamma_{j}$ exactly at the points of $\Gamma_{j+1}$, whereas $l$ is nowhere tangent to $\Gamma_{s}$. A local model of this situation is given by the following:

$$
\begin{aligned}
\Omega & =\left\{x \in \mathbb{R}^{n}: x_{1}>0\right\}, \quad \Gamma=\left\{x_{1}=0\right\}, \\
l & =x_{2} \partial_{1}+x_{3} \partial_{2}+\ldots+x_{k} \partial_{k-1}+\partial_{k}, \quad k \leq n, \\
\Gamma_{j} & =\left\{x_{1}=x_{2}=\ldots=x_{2+j}=0\right\}, \quad j=0, \ldots, k-2 .
\end{aligned}
$$

Some function spaces of the right-hand side and solutions ensuring the unique solvability of the problem are found in [59]. The success was achieved by a choice of weight functions in the derivation of a priori estimates for the solution. Additionally, I proved that the inverse operator of the problem is always compact on $L^{p}(\Gamma), 1<p \leq \infty$. It turned out that the tangent manifolds of codimension greater than one do not influence the correct statement of the problem, contrary to Arnold's expectations.

However, the following problem is still unsolved.

Problem 39. Describe the asymptotic behaviour of solutions near the points of tangency of the field $l$. In particular, it seems challenging to find asymptotics near the point of $\Gamma_{1}$ whose neighbourhood contains both points of entrance and exit of the field $l$ with respect to $\Gamma$.

More generally, it would be interesting to obtain regularity results for strong solutions of the generic degenerating problem. 


\subsection{Matrix Generalization of the Oblique Derivative Problem}

Let $\Omega$ be a domain in $\mathbb{R}^{n}$. Consider the Laplace equation $\Delta \mathbf{u}=0$ in $\Omega$, where $\mathbf{u}$ is a $k$-dimensional vector field, and add the condition

$$
\sum_{i=1}^{n} A_{i}(x) \frac{\partial \mathbf{u}}{\partial x_{i}}=\varphi(x) \quad \text { on } \partial \Omega,
$$

where $\left\{A_{i}\right\}_{1 \leq i \leq n}$ is a collection of $k \times k$ matrix function.

Problem 40. Develop a theory of solvability of the boundary value problem above in the case when the corresponding pseudodifferential operator on $\partial \Omega$ is not elliptic. An interesting particular case is the boundary condition

$$
(\mathbf{a}+i \mathbf{b}) \cdot \nabla u=\varphi,
$$

where $\mathbf{a}$ and $\mathbf{b}$ are real vector fields and $u$ is a complex-valued scalar function.

\subsection{Coercive Weighted Estimates for Elliptic Equations in Domains with Inner Cusps}

Consider the Dirichlet problem for an arbitrary elliptic equation of order $2 m$ in a domain $\Omega \subset \mathbb{R}^{n}$ with inner cusp. Two-sided estimates for derivatives of order $2 m$ of solutions were obtained in [32, Chapter 10] with $n>2 m+1$.

Problem 41. Obtain estimates of the same nature for $n \leq 2 m+1$.

\subsection{Multiplicative Inequality for the Linear Elasticity System in an Arbi- trary Domain}

One of the theorems obtained in [47] reads as follows:

Let $\Omega$ be a three-dimensional domain and let $\mathcal{L}$ denote the Lamé operator defined by

$$
\mathcal{L} u=-\Delta u-\alpha \nabla \operatorname{div} u,
$$

where $\alpha=1 /(1-2 \nu)>-1$ and $\nu$ is the Poisson's ratio. Let $1<q<3$, $p=q /(q-1)$ and let $L_{0}^{1, q}(\Omega)$ stand for the completion of $C_{0}^{\infty}(\Omega)$ in the Sobolev space $L^{1, q}(\Omega)$. By [47], the condition

$$
\alpha \in\left(\alpha_{-}, \alpha_{+}\right) \approx(-0.194,1.524),
$$

implies the inequality

$$
\|u\|_{L^{\infty}(\Omega)} \leq C\|\mathcal{L} u\|_{L^{p}(\Omega)}^{1 / 2}\|\nabla u\|_{L^{q}(\Omega)}^{1 / 2},
$$

where $C$ is an absolute constant depending only on $\alpha$.

Problem 42. Does (5.17) hold for an arbitrary domain $\Omega \subset \mathbb{R}^{3}$ without the assumption (5.16)?

\subsection{2. $L^{p}$-Dissipativity of the Lamé Operator}

Consider the Dirichlet problem with zero boundary condition for the Lamé operator $\mathcal{L}$ defined in the previous section. 
We say that $\mathcal{L}$ is $L^{p}$-dissipative if for all $u \in\left[C_{0}^{\infty}(\Omega)\right]^{n}$

$$
\int_{\Omega} \mathcal{L} u \cdot|u|^{p-2} u d x \leq 0
$$

where $1<p<\infty$.

In [9, Chapter 3], we prove that for $n=2$ the operator $\mathcal{L}$ is $L^{p}$-dissipative if and only if

$$
\left(\frac{1}{2}-\frac{1}{p}\right)^{2} \leq \frac{2(\nu-1)(2 \nu-1)}{(3-4 \nu)^{2}} .
$$

For $n=3$ this inequality is necessary for the $L^{p}$-dissipativity of $\mathcal{L}$ and there are some sufficient conditions. However, the following problem is unsolved.

Problem 43. Prove or disprove that (5.18) is sufficient for the $L^{p}$-dissipativity of $\mathcal{L}$ in the three-dimensional case.

\subsection{Generalized Maximum Principle for the Lamé System in a Domain with Non-Lipschitz Graph Conical Vertex}

Let $\Omega$ be a domain with compact closure and whose boundary $\partial \Omega$ is smooth outside one point. We assume that near this point $\partial \Omega$ coincides with a cone, not necessarily a Lipschitz graph.

We consider the Dirichlet problem

$$
\begin{aligned}
\Delta \mathbf{u}+(1-2 \nu)^{-1} \nabla \operatorname{div} \mathbf{u} & =0 & & \text { in } \Omega, \\
\mathbf{u} & =\mathbf{g} & & \text { on } \partial \Omega,
\end{aligned}
$$

where $\nu<1$. The case $\nu=1 / 2$ corresponds to the Stokes system, when the condition $\operatorname{div} \mathbf{u}=0$ becomes explicit.

By [81] for $n=3$ (see also [33, Sections 3.6 and 5.5]) and [13] for $n \geq 3$, the generalized maximum principle

$$
\|\mathbf{u}\|_{C(\bar{\Omega})} \leq C\|\mathbf{g}\|_{C(\partial \Omega)}
$$

holds provided $\nu \leq 1 / 2$. The proof fails for $\nu>1 / 2$, which gives rise to the following

Problem 44. Prove (5.19) under the assumption $1 / 2<\nu<1$.

\subsection{Boundedness of the Displacement Vector in the Traction Problem in} Domains with Non-Lipschitz Graph Conical Vertices

Let $\Omega$ be a domain in $\mathbb{R}^{3}$ with $\partial \Omega$ smooth outside one point, the vertex of a non-Lipschitz graph cone. Consider the Neumann problem for the linear elasticity system

$$
\begin{gathered}
\frac{\partial \sigma_{i, j}(\mathbf{u})}{\partial x_{j}}=f \quad \text { in } \Omega, \\
\sigma_{i, j}(\mathbf{u}) \nu_{j}=0 \quad \text { on } \partial \Omega,
\end{gathered}
$$


where $f \in C_{0}^{\infty}(\Omega), \mathbf{u}$ is the displacement vector,

$$
\begin{aligned}
\sigma_{i, j} & =\lambda \theta \delta_{i, j}+2 \mu \varepsilon_{i, j}, \quad \theta=\varepsilon_{11}+\varepsilon_{22}+\varepsilon_{33}, \\
\varepsilon_{i, j} & =\frac{1}{2}\left(\frac{\partial u_{i}}{\partial x_{j}}+\frac{\partial u_{j}}{\partial x_{i}}\right) .
\end{aligned}
$$

Problem 45. Prove or disprove that the displacement vector is uniformly bounded.

In case of Lipschitz graph domains, the positive answer was given in $[12,29]$, and [11].

\subsection{Comparison of Martin's and Euclidian Topologies}

In [65] a description of the Martin boundary and of the minimal positive harmonic functions were given without proofs for a class of domains in $\mathbb{R}^{n}$. The description just mentioned is as follows.

Let $\Omega$ be a bounded domain in $\mathbb{R}^{n}$ with Euclidean boundary $\partial \Omega, O \in$ $\partial \Omega$, and let $\partial \Omega \backslash\{O\}$ be a $C^{2}$ manifold. Take spherical coordinates $(\rho, \tau, \alpha)$ with origin $O\left(\rho \geq 0,|\tau| \leq \pi / 2, \alpha \in S^{n-2}\right)$ and consider the case when $\Omega$ is obtained by rotating about the axis $|\tau|=\pi / 2$ a domain $\omega$ contained in the half-plane $\{(\rho, \tau): \rho>0,|\tau|<\pi / 2\}$, which is given near $\rho=0$ by an inequality of the form $|\tau-\psi(\rho)|<\Theta(\rho)$, so that the boundary of $\Omega$ near $O$ is made up of two tangential components under certain conditions on $\psi$ and $\Theta$. The necessary and sufficient condition for $O$ to give rise to exactly one Martin boundary point is the divergence of the integral

$$
\int_{0}^{1} \frac{\Theta(t) d t}{(\pi-2|\psi(t)|)^{2} t} .
$$

In other words, (5.21) is equivalent to the existence of the limit

$$
\lim _{x \rightarrow 0} \frac{G(x, y)}{G(x, z)},
$$

where $G$ is Green's function and $x, y, z$ are points in $\Omega$.

For me, the period of time when I worked on the topic, was difficult and I was not able to prepare a detailed exposition. Even later I did not return to the area so that [65] became a collection of unproved lemmas. Since it is impossible for me to do this job in the future, I dare to propose it as an unsolved

Problem 46. Justify the criterion (5.21) and prove assertions on the Martin boundary formulated in [65].

\section{Nonlinear Elliptic Operators}

\subsection{Nonlinear Singularities at the Vertex of a Cone}

Let $\Delta_{p}$ be the $p$-Laplace operator

$$
u \rightarrow \operatorname{div}\left(|\nabla u|^{p-2} \operatorname{grad} u\right) .
$$


Variational solutions of the Dirichlet problem for the $p$-Laplace equation in a cone $\Omega \subset \mathbb{R}^{n}$ with vertex at the origin $O \in \mathbb{R}^{n}$ :

$$
\begin{aligned}
\Delta_{p} u=0 & \text { in } \Omega \text { near } O, \\
u=0 & \text { on } \partial \Omega \text { near } O
\end{aligned}
$$

admit the asymptotics

$$
u(x) \sim C|x|^{\lambda} \Psi\left(\frac{x}{|x|}\right)
$$

see [37] and [103].

Problem 47. No analogue of (6.1) is known for higher order nonlinear equations

$$
\sum_{|\alpha| \leq m} \partial^{\alpha}\left(\mathbf{a}_{\alpha}\left(\nabla_{m} u\right)\right)=0
$$

where $\mathbf{a}_{\alpha}$ are positive homogeneous vector-valued functions. The same applies elliptic systems of the second order.

\subsection{Nonlinear Boundary Value Problem with Infinite Dirichlet Data}

Let $\Omega$ be a bounded smooth domain in $\mathbb{R}^{n}$ and let $Q$ denote a function on $\Omega \times \mathbb{R}^{n}$, positive homogeneous of degree $p \in(1, \infty), Q(x, \xi)>0$ for $x \in \Omega$, $\xi \neq 0$. By $\Delta_{p}$ we denote the $p$-Laplace operator.

Problem 48. Prove that there exists a unique positive $\lambda$ such that the Dirichlet problem

$$
\begin{aligned}
\Delta_{p} u+Q(x, \nabla u)+\lambda & =0 \quad \text { in } \quad \Omega, \\
\left.u\right|_{\partial \Omega} & =-\infty
\end{aligned}
$$

is uniquely solvable up to a constant term.

Remark. The pair $(u, \lambda)$ should solve the variational problem

$$
\lambda=\sup _{\left.v\right|_{\partial \Omega}=-\infty} \inf _{x \in \Omega}\left(-\Delta_{p} v-Q(x, \nabla v)\right) .
$$

It is obvious that for $p=2$ and $Q(\xi)=|\xi|^{2},(6.2)$ is the classical variational principle for the first eigenvalue of the Laplacian [96].

\subsection{Singularities of Solutions to the Neumann Problem for a Semilinear Equation}

Let the planar domain $\Omega$ have an angle with vertex $O$. The Neumann problem

$$
\left\{\begin{array}{l}
\Delta u+a(x) u_{x_{1}}^{2}+2 b(x) u_{x_{1}} u_{x_{2}}+c(x) u_{x_{2}}^{2}=0 \quad \text { in } \Omega, \\
\frac{\partial u}{\partial n}=0 \quad \text { on } \partial \Omega \text { near } O,
\end{array}\right.
$$

is considered in [28], where an asymptotic formula for an unbounded solution is given under the assumption of the positivity of the quadratic form

$$
a(x) \xi^{2}+2 b(x) \xi \eta+c(x) \eta^{2} .
$$


Roughly speaking, the solution has a $\log \log r^{-1}$ singularity. This asymptotic behaviour fails without positivity of the quadratic form. Indeed, the function $u(x)=\log r^{-1}+\cos \theta$ satisfies

$$
\Delta u+\frac{\cos \theta}{1+\sin ^{2} \theta}|\nabla u|^{2}=0
$$

on $\mathbb{R}_{+}^{2}$ as well as zero Neumann condition. This example suggests the following problem.

Problem 49. Describe the asymptotic behaviour of solutions to (6.3) without assumption of positivity of the quadratic form (6.4).

\subsection{Positive Solutions of a Non-Linear Dirichlet Problem with Zero Bound- ary Data}

Let $\Omega$ be a bounded planar domain and let $\partial \Omega$ be smooth except for an angular point $O$.

Problem 50. Study the existence, uniqueness and regularity of solutions of the equation

$$
\Delta u-\lambda u^{k}=0 \quad \text { in } \Omega
$$

that are positive in $\Omega$ and vanish on $\partial \Omega \backslash\{O\}$. Here $\lambda$ and $k$ are real numbers. Remark. Obviously, for $\lambda=0$ and the sector

$$
\Omega=\{x=r \exp (i \varphi): 0<r<1,0<\varphi<\alpha\}
$$

all solutions in question are given by

$$
u(x)=C\left(r^{-\pi / \alpha}-1\right) \sin \left(\frac{\pi}{\alpha} \varphi\right) .
$$

\subsection{Positive $p$-Harmonic Functions in a Cone}

Let $u$ denote a positive locally bounded solution of the equation $\Delta_{p} u=0$ in a cone vanishing at the boundary.

Problem 51. Prove that

$$
u(x)=C|x|^{\lambda} \psi\left(\frac{x}{|x|}\right),
$$

where $\lambda>0$ and $C$ is an arbitrary constant.

The existence of solutions appearing in the right-hand side of (6.5) was studied in [37] and [103]. If the cone is the half-space $\left\{x: x_{n}>0\right\}$, formula (6.5) becomes $u(x)=C x_{n}$.

\subsection{Phragmen-Lindelöf Principle for the $p$-Laplace equation}

By [58], bounded solutions $u(x)$ of the $p$-Laplace equation, $1<p<n$, with zero Dirichlet data in a $\delta$-neighborhood of a point $O \in \partial \Omega$, admit the pointwise majorant

$$
C \exp \left(-c \int_{|x|}^{\delta}\left(\frac{\operatorname{cap}_{p}\left(B_{\rho} \backslash \Omega\right)}{\operatorname{cap}_{p}\left(B_{\rho}\right)}\right)^{1 /(p-1)} \frac{d \rho}{\rho}\right),
$$

where

$$
\operatorname{cap}_{p}(F)=\inf \left\{\|\nabla u\|_{L^{p}\left(\mathbb{R}^{n}\right)}^{p}: u \in C_{0}^{\infty}\left(\mathbb{R}^{n}\right), u \geq 1 \text { on } F\right\} .
$$


Problem 52. Prove the following conjecture. If the energy integral of $u$ is divergent, then, for $2 r<\delta$,

$$
\|u\|_{L^{p}\left(B_{\delta} \backslash B_{r}\right)} \geq \frac{c(\delta)}{r^{\frac{n-p}{p-1}}} \exp \left(c \int_{r}^{\delta} \frac{\operatorname{cap}_{p}\left(B_{\rho} \backslash \Omega\right)}{\operatorname{cap}_{p}\left(B_{\rho}\right)} \frac{d \rho}{\rho}\right) .
$$

This assertion was established for the linear uniformly elliptic equation

$$
-\frac{\partial}{\partial x_{i}}\left(a_{i j}(x) \frac{\partial u}{\partial x_{j}}\right)=0
$$

with measurable bounded coefficients in [55].

\subsection{Isolated Singularity of Solution to a Nonlinear Elliptic System}

In [16], J. Frehse noticed that the elliptic system with smooth nonlinearity

$$
\begin{aligned}
& \Delta u_{1}=-\frac{2 u_{1}}{|\mathbf{u}|^{2}+1}\left(\left|\frac{\partial \mathbf{u}}{\partial x}\right|^{2}+\left|\frac{\partial \mathbf{u}}{\partial y}\right|^{2}\right), \\
& \Delta u_{2}=-\frac{2 u_{2}}{|\mathbf{u}|^{2}+1}\left(\left|\frac{\partial \mathbf{u}}{\partial x}\right|^{2}+\left|\frac{\partial \mathbf{u}}{\partial y}\right|^{2}\right)
\end{aligned}
$$

has the weak discontinuous solution

$$
u_{1}=\cos \log r, \quad u_{2}=\sin \log r,
$$

where $r=\sqrt{x^{2}+y^{2}}<e^{-2}$.

Problem 53. Is the right-hand side of (6.8) the only possible isolated singularity of solutions to system (6.7) at the origin?

\subsection{Poisson Type Solutions of Riccati's Equation with Dirichlet Data}

Let $\Omega$ be a smooth, bounded planar domain and let the origin $O$ be a point of $\partial \Omega$. Consider the equation

$$
\Delta u+\alpha u_{x}^{2}+\beta u_{y}^{2}=0 \quad \text { in } \Omega
$$

where $\alpha$ and $\beta$ are smooth positive functions given on $\bar{\Omega}$. We assume that the solution $u$ satisfies $u=0$ on $\partial \Omega \backslash\{O\}$.

Problem 54. Prove that either $u=0$ on $\partial \Omega$, or $u$ has a logarithmic singularity at $O$ and depends upon an arbitrary constant.

A formal asymptotic expansion of $u$ near $O$ can be found in [92], also for more general equations.

\section{Parabolic, Hyperbolic and General Differential Operators}

\subsection{Behaviour at Infinity of Solutions to the Cauchy-Dirichlet Problem for} a Semi-Linear Parabolic Equation

Consider the equation

$$
\frac{\partial u}{\partial t}-\Delta u=\nabla u A(x) \nabla u,
$$


in the half-cylinder $\{(x, t): x \in \Omega, t>0\}$, where $A$ is a positive-definite matrix and $\Omega$ is a bounded domain in $\mathbb{R}^{n}$. Assume that

$$
u=0 \text { for } x \in \partial \Omega, t>0 .
$$

If $A$ is the unit matrix, the replacement of $u$ by $\log v$ with $v>0$ shows that at infinity either $u$ vanishes exponentially, or $u$ behaves as $t$ at any positive distance from the boundary.

Problem 55. Show that the same alternative holds if $A$ is not the unit matrix. Describe an asymptotic behaviour of unbounded solutions. What happens if $A$ is not positive-definite?

\subsection{Two Inequalities Related to the Heat Equation}

Let $\Omega$ be an open subset of $\mathbb{R}^{n}$. By [49] and [71, Section 2.5.2], the inequality

$$
\int_{\Omega}|u|^{2} d \mu \leq C \int_{\Omega}|\nabla u|^{2} d x
$$

where $\mu$ is a non-negative measure in $\Omega$, holds for all $u \in C_{0}^{\infty}(\Omega)$ if and only if

$$
\sup _{F} \frac{\mu(F)}{\operatorname{cap}(F, \Omega)}<\infty \text {. }
$$

Here $\operatorname{cap}(F, \Omega)$ is the relative harmonic capacity of a compact subset $F$ of $\Omega$ with respect to $\Omega$

$$
\operatorname{cap}(F, \Omega)=\inf \left\{\int_{\Omega}|\nabla v|^{2} d x: v \in C^{0,1}(\Omega), v \geq 1 \text { on } F\right\} .
$$

Inequality (7.1) is important in particular in the theory of the Schrödinger operator $\Delta+\mu$, see [49] and [71, Section 2.5].

The following inequality finds applications in the theory of the CauchyDirichlet problem for the operator

$$
u \rightarrow \frac{\partial u}{\partial t}-\Delta u-\mu u
$$

in the cylinder $\Omega \times(0, T)$, where $\mu$ is a measure defined on this cylinder:

$$
\int_{\Omega \times[0, T)} u^{2} d \mu(x, t) \leq C\left(\int_{\Omega \times[0, T)}\left|\nabla_{x} u\right|^{2} d x d t+\sup _{t \in[0, T)} \int_{\Omega}(u(x, t))^{2} d x\right) .
$$

Problem 56. Give a complete characterization of the measure $\mu$ subject to the inequality (7.3) for all smooth functions u given on $\Omega \times[0, T)$ and vanishing near $\partial \Omega \times[0, T]$.

Answer the same question for the inequality

$$
\int_{\Omega \times[0, T)} u^{2} d \mu(x, t) \leq C\left(\int_{\Omega \times[0, T)}\left|\nabla_{x} u\right|^{2} d x d t+\int_{0}^{T}\left\|\frac{\partial u}{\partial t}\right\|_{L^{-1,2}(\Omega)}^{2} d t\right),
$$

where $L^{-1,2}(\Omega)$ is the dual space of $L_{0}^{1,2}(\Omega)$.

A necessary condition of type (7.2) obviously holds with the parabolic capacity studied in [97]. Most probably, it is also sufficient. 


\subsection{Trace Space for the Maximal Operator Generated by the Wave Operator}

Let $\Omega$ be a domain in $\mathbb{R}^{1} \times \mathbb{R}^{n}$ and let $\square_{x, t}=\partial^{2} / \partial t^{2}-\Delta_{x}$. By $\square$ denote the maximal operator generated by $\square_{x, t}$, i.e. the closure of $\square_{x, t}$ defined on $C^{\infty}(\bar{\Omega})$ in the norm

$$
\left(\left\|\square_{x, t} u\right\|_{L^{2}(\Omega)}^{2}+\|u\|_{L^{2}(\Omega)}^{2}\right)^{1 / 2}
$$

Problem 57. Describe the space of boundary traces for functions in the domain of $\bar{\square}$.

Remark. If $\Omega=\mathbb{R}_{+}^{n+1}=\left\{(x, t): x \in \mathbb{R}^{n}, t>0\right\}$, then by [18, Corollary 4.6.2] the traces in question make up the Besov space $B^{1 / 2,2}\left(\mathbb{R}^{n}\right)$.

\subsection{Characteristic Problem for Nonlinear Hyperbolic Operators}

In [106], the authors study the initial value problem for a hyperbolic linear equation of order $2 m$, when the initial surface has characteristic points on some compact set $\Gamma$. It is proved that the problem is well posed if the set $\Gamma$ is free from the last initial condition $D_{t}^{2 m-1} u=f$. In the case $m=1$ a similar result was later obtained in [22].

Problem 58. Obtain a similar result without linearity assumption for the hyperbolic operator.

The only result in this direction known to me is in [6]. It concerns the global characteristic Cauchy problem (Goursat problem) for the nonlinear wave equation. The boundary data in [6] are prescribed on the light cone with two singularities representing both past and future.

\subsection{Boundary Traces of Functions in the Domain of a Maximal Differential Operator with $\boldsymbol{t}$-Dependent Coefficients}

Let $P\left(D_{t}, D_{x}\right)$ be an arbitrary differential operator acting on functions defined on the half-space $\mathbb{R}_{+}^{n}=\left\{(x, t): x \in \mathbb{R}^{n}, t>0\right\}$ and let $P_{\max }$ be the corresponding maximal operator. The domain of $P_{\max }$ is the completion of the space of functions smooth on $\overline{\mathbb{R}_{+}^{n}}$ and vanishing at infinity in the norm

$$
\|P u\|_{L^{2}\left(\mathbb{R}_{+}^{n}\right)}+\|u\|_{L^{2}\left(\mathbb{R}_{+}^{n}\right)} .
$$

In [18, Chapter 2], an algebraic characterization of boundary traces of functions in the domain of $P_{\max }$ was found.

Problem 59. Describe traces on the hyperplane $t=0$ of functions in the domain of $P_{\max }$ assuming that $P$ is an arbitrary differential operator of the first order in $t$ with coefficients depending on $t$.

This is only an example which points to the possible development of the theory in [18] for differential operators with coefficients depending only on $t$. 


\subsection{Differential Operators Acting in Pairs of Sobolev Spaces}

Obviously, a differential operator of order $h$ with variable coefficients maps the Sobolev space $L^{l, p}\left(\mathbb{R}^{n}\right)$ into $L^{l-h, p}\left(\mathbb{R}^{n}\right)$ if the coefficients are multipliers in the proper pairs of Sobolev spaces. This statement can be inverted for some classes of differential operators as shown in [88, Section 10.1.1]. However, the following counterexample proposed in [88, Section 10.1.2] shows that this is not a general property of differential operators.

The coefficient $a$ of the operator

$$
u \rightarrow a(x) \frac{\partial u}{\partial x_{n}}: W^{2,2}\left(\mathbb{R}^{n}\right) \rightarrow L^{2}\left(\mathbb{R}^{n}\right),
$$

where $W^{l, 2}=L^{l, 2} \cap L^{2}$, need not be a multiplier from $W^{1,2}\left(\mathbb{R}^{n}\right)$ into $L^{2}\left(\mathbb{R}^{n}\right)$. This gives rise to the following

Problem 60. Find a condition on the function a, necessary and sufficient for the validity of the inequality

$$
\left\|a(x) \frac{\partial u}{\partial x_{n}}\right\|_{L^{2}\left(\mathbb{R}^{n}\right)} \leq c\|u\|_{W^{2,2}\left(\mathbb{R}^{n}\right)}
$$

for all $u \in C_{0}^{\infty}\left(\mathbb{R}^{n}\right)$, where $n>3$.

Let me point out that if $a$ is independent of the variable $x_{n}$, then the criterion is

$$
\sup _{0<r<1, y^{\prime} \in \mathbb{R}^{n-1}} r^{3-n} \int_{\left|x^{\prime}-y^{\prime}\right|<r} a\left(x^{\prime}\right) d x^{\prime}<\infty,
$$

see [88, Section 10.1.2].

\subsection{Existence of a Well-Posed Boundary Value Problem for for General Differential Operators in $L^{p}(\Omega)$}

Hörmander (1955) showed the existence of a well-posed boundary value problem for every differential operator with constant coefficients in an arbitrary bounded domain. Equivalently, he proved the estimate

$$
\|u\|_{L^{2}(\Omega)} \leq C\|\mathcal{L}(D) u\|_{L^{2}(\Omega)},
$$

where $u$ is an arbitrary function in $C_{0}^{\infty}(\Omega)$.

Problem 61. Does (7.6) hold with $L^{p}(\Omega)$ instead of $L^{2}(\Omega)$ ?

\subsection{Existence of a Well-Posed Boundary Value Problem for Unbounded Do- mains}

Estimate (7.6) fails for some unbounded domains which gives rise to

Problem 62. Let $\mathcal{L}(D)$ be a differential operator in $\mathbb{R}^{n}$ with constant coefficients. Characterise the domains satisfying (7.6).

Example. For $\mathcal{L}(D)=\Delta-1$, any domain is admissible whereas for $\mathcal{L}(D)=\Delta$, $n=3$, inequality (7.6) holds if and only if $\Omega$ does not contain balls with arbitrary radii. For $n \geq 4$, one needs the notion of biharmonic capacity to formulate a necessary and sufficient condition in terms of a capacitary inner radius (see [71, Chapter 16]). 
One can even ask the same question for special operators. For instance, it seems interesting to characterize unbounded domains for which the inequalities

$$
\begin{aligned}
\|u\|_{L^{p}(\Omega)} & \leq C\|\Delta u+u\|_{L^{p}(\Omega)}, \\
\|u\|_{L^{p}(\Omega)} & \leq C\|\square u\|_{L^{p}(\Omega)}, \\
\|u\|_{L^{p}(\Omega)} & \leq C\left\|\partial_{x_{n}} u-\Delta_{x^{\prime}} u\right\|_{L^{p}(\Omega)}
\end{aligned}
$$

hold for all $u \in C_{0}^{\infty}(\Omega)$. Here $\square$ and $\partial_{x_{n}}-\Delta_{x^{\prime}}$ are the wave and heat operators.

\section{Stationary Stokes and Navier-Stokes Systems}

\subsection{Hölder Regularity of Solutions to the $n$-Dimensional Dirichlet-Stokes Problem}

Consider the Dirichlet problem for the Stokes system

$$
\begin{aligned}
-\nu \Delta \mathbf{v}+\nabla p=\mathbf{f} & & \text { in } \Omega, \\
\operatorname{div} \mathbf{v}=0 & & \text { in } \Omega, \\
\mathbf{v}=\mathbf{g} & & \text { on } \partial \Omega,
\end{aligned}
$$

where $\Omega$ is a polyhedron in $\mathbb{R}^{n}, n \geq 3$, and $\mathbf{v} \in L^{1,2}(\Omega)$.

Problem 63. Assuming that $\mathbf{g}$ belongs to the Hölder space $C^{0, \alpha}(\partial \Omega)$ for a certain $\alpha \in(0,1)$, prove that there exists $\beta \in(0,1)$ such that $\mathbf{v} \in C^{0, \beta}(\bar{\Omega})$.

Remark. The starting point could be the following property obtained in [13]: for any solution of the form $|x|^{\lambda} \psi(x /|x|)$ with $\operatorname{Re} \lambda>1-n / 2$ one has $\operatorname{Re} \lambda>0$.

\subsection{Differentiability of Solutions to the Dirichlet Problem for the Equations of Hydrodynamics in Convex Domains}

Consider the Dirichlet problem for the Stokes system

$$
\begin{aligned}
-\nu \Delta \mathbf{v}+\nabla p=\mathbf{f} & & \text { in } \Omega, \\
\operatorname{div} \mathbf{v}=0 & & \text { in } \Omega, \\
\mathbf{v}=\mathbf{g} & & \text { on } \partial \Omega .
\end{aligned}
$$

The following result is obtained in [30, Section 6.3].

Let $\Omega$ be a bounded convex two-dimensional domain and let $\mathbf{f} \in L^{q}(\Omega)$, for some $q>2$. Then $\mathbf{v} \in C^{0,1}(\bar{\Omega})$, i.e. $\mathbf{v}$ is Lipschitz and

$$
\|\mathbf{v}\|_{C^{0,1}(\bar{\Omega})} \leq C\|\mathbf{f}\|_{L^{q}(\Omega)},
$$

where $C$ depends only on $\Omega$.

The following similar result for the Navier-Stokes system was also established in [30, Section 6.3].

Let $L_{0}^{1,2}$ be the space introduced in Sect. 3.4. Suppose that $(\mathbf{v}, p) \in$ $L_{0}^{1,2}(\Omega) \times L^{2}(\Omega)$ satisfy the Dirichlet problem

$$
\begin{aligned}
-\nu \Delta \mathbf{v}+\nabla p+\sum_{1 \leq k \leq 2} v_{k} \partial_{x_{k}} \mathbf{v}=\mathbf{f} & & \text { in } \Omega \\
\operatorname{div} \mathbf{v}=0 & & \text { in } \Omega \\
\mathbf{v}=0 & & \text { on } \partial \Omega
\end{aligned}
$$


where $\Omega$ is a bounded convex two-dimensional domain and $\mathbf{f} \in L^{q}(\Omega)$ for some $q>2$. Then $\mathbf{v} \in C^{0,1}(\bar{\Omega})$.

Problem 64. Show that the above two facts hold with $q>2$ replaced by $q>3$ for the three-dimensional case.

\subsection{Boundedness of Solutions to the Dirichlet Problem for the Stokes Sys- tem in Arbitrary 3D Domains}

Let $\Omega$ be an arbitrary bounded three-dimensional domain. Consider the Dirichlet problem (8.1).

Problem 65. Prove or disprove that the variational solutions are uniformly bounded in $\Omega$.

\subsection{Resolvent $L^{p}$-Estimates for the Dirichlet-Stokes Operator}

Let $R_{\lambda}$ be the resolvent operator of the Dirichlet problem

$$
\begin{aligned}
\Delta \mathbf{v}+\nabla p+\lambda \mathbf{v}=\mathbf{f} & & \text { in } \Omega, \\
\operatorname{div} \mathbf{v}=0 & & \text { in } \Omega, \\
\mathbf{v}=0 & & \text { on } \partial \Omega,
\end{aligned}
$$

where $\lambda \in \mathbb{R}^{1}$ and $\Omega$ is a bounded domain in $\mathbb{R}^{n}$.

Problem 66. 1 . Justify the following conjecture. If $\Omega \subset \mathbb{R}^{n}$ is convex, then, for all $\lambda \in \mathbb{R}^{1} \backslash\{0\}$ and $1<p<\infty$,

$$
\left\|R_{\lambda}\right\|_{L^{p}(\Omega) \rightarrow L^{p}(\Omega)} \leq \frac{c}{|\lambda|},
$$

where $c$ is a constant which does not depend on $\lambda$.

2. Let $\Omega$ be smooth outside of a smooth $(n-2)$-dimensional submanifold $M$ without boundary. Assume that $\partial \Omega$ has a reentrant dihedral angle with edge $M$ and opening $\alpha>\pi$. Prove that (8.2) holds for all $\lambda \in \mathbb{R}^{1} \backslash\{0\}$ if

$$
\left|\frac{1}{p}-\frac{1}{2}\right|<\frac{\pi}{2 \alpha} .
$$

Note that for three-dimensional Lipschitz graph domains the question of validity of (8.2) for

$$
\frac{3}{2}-\varepsilon<p<3+\varepsilon
$$

with certain $\varepsilon>0$, was raised by Taylor [102] and answered affirmatively by Shen [100].

\subsection{Non-Uniqueness for the Stationary Navier-Stokes System with Dirichlet Data}

Let $\Omega$ be a simply connected bounded domain in $\mathbb{R}^{3}$. It is well known [40] that the Dirichlet problem for the stationary Navier-Stokes system has at most one variational solution provided the Reynolds number is sufficiently small. It seems probable that the uniqueness fails for large Reynolds numbers.

Problem 67. Construct a counterexample showing that the just mentioned Dirichlet problem may have more than one solution for small viscosity and big data. 
This problem is classical but seems half-forgotten to me. Therefore, I include it in the present list.

\section{Theory of Surface Waves}

\subsection{Well-Posed Neumann-Kelvin Problem for a Surface-Piercing Body}

When solving the seakeeping problem with forward speed which is called Neumann-Kelvin problem, one looks for a velocity potential $u(x, y, z)$ defined on a lower half-space outside the wetted part $S$ of a body moving in the $x$ direction. The normal derivative of $u$ is prescribed on $S$ and the condition

$$
\frac{\partial^{2} u}{\partial x^{2}}+\nu \frac{\partial u}{\partial z}=0, \quad \nu=\text { const }>0
$$

should be satisfied on the flat surface $F$ outside the body (see [38, Chapter 8]).

This classical boundary value problem is in an unsatisfactory state both from theoretical and numerical points of view.

Problem 68. Verify the following conjecture. The Neumann-Kelvin problem becomes Fredholm if the above formulation is completed by prescribing the elevation of the free surface on a part of the water line $L$, to be more precise, by adding the values of $\partial u / \partial x$ at those points of $L$ where the angle between the exterior normal to $L$ and the $x$-axis is not greater than $\pi / 2$.

Note that the asymptotics of $u$ near the curve $L$ is not known. However, the existence of $\partial u / \partial x$ is suggested by the asymptotic formula (8.8) in [38] obtained for the two-dimensional case.

\subsection{Solvability of the Two-Dimensional Kelvin-Neumann Problem for a Submerged Cylinder}

In [46], the two-dimensional problem on the steady flow of infinite depth about a submerged cylinder is considered and the existence of the unique solution of any velocity $v$ of the undisturbed flow upstream in the case of an arbitrary circular cylinder is proved.

This boundary value problem is stated as follows. One looks for the velocity potential $u(x, y)$ of the steady motion of a heavy ideal incompressible fluid induced by the cylinder with the cross-section $\Omega$ which moves uniformly in the $x$-direction. The function $u$ satisfies

$$
\begin{aligned}
\Delta u=0 & \text { in } W=\mathbb{R}_{-}^{2} \backslash \bar{\Omega}, \\
\frac{\partial^{2} u}{\partial x^{2}}+\nu \frac{\partial u}{\partial y}=0 & \text { for } y=0,
\end{aligned}
$$

where $\mathbb{R}_{-}^{2}=\{(x, y): y<0\}$ and $\nu=g v^{-2}$ with $g$ being the acceleration due to gravity. In addition,

$$
\begin{array}{r}
\sup |\nabla u|<\infty, \quad|\nabla u|=\mathrm{o}(1) \quad \text { as } x \rightarrow \infty, \\
\frac{\partial u}{\partial n}=v \cos (N, x) \quad \text { on } \partial \Omega,
\end{array}
$$

where $N$ is the unit normal to $\partial \Omega$ directed into $\Omega$. 
The second relation in (9.3) is equivalent to the absence of waves far upstream.

The result obtained in [46], which is of course rather special, seems, however, to be the only known uniqueness theorem for the boundary value problem just stated not relying on any restrictions on $\nu$.

Hence we naturally arrive at the following

Problem 69. Show the unique solvability of (9.1)-(9.4) for a fairly arbitrary domain and for all values of $\nu$.

Note that the proof in [46] is based on the theorem in [105] ensuring the uniqueness of a solution with finite Dirichlet integral under the assumption

$$
x \cos (N, x) \geq 0 \quad \text { on } \partial \Omega .
$$

The proof of uniqueness in [105] concerns the $n$-dimensional case, $n \geq 2$.

For a modern state of the art presentation of the linear theory of water waves see [38].

\subsection{Counterexample in the Water-Wave Problem for a Submerged Body}

Let us use the geometrical assumptions made in Section 48. The boundary value problem of harmonic oscillations of the fluid induced by a submerged body is stated as follows. The function $u$ satisfies (9.1), (9.3), while (9.2) is replaced by

$$
\frac{\partial u}{\partial y}-\nu u=0 \quad \text { for } y=0
$$

and a radiation condition at infinity is required (see [38]).

In [64] a class of domains is found for which the problem in question is uniquely solvable for all $\nu>0$, see also [38, Chapter 1].

Problem 70. Construct an example of a connected set $\Omega$ showing that some restrictions on $\Omega$ ensuring solvability are necessary.

\subsection{Sharp Hardy-Leray Inequality for Divergence-Free Fields}

Let $\mathbf{u}$ denote a vector field in $\mathbb{R}^{n}$ with components in $C_{0}^{\infty}\left(\mathbb{R}^{n}\right)$. The following $n$-dimensional generalization of the one-dimensional Hardy inequality [21]

$$
\int_{\mathbb{R}^{n}} \frac{|\mathbf{u}|^{2}}{|x|^{2}} d x \leq \frac{4}{(n-2)^{2}} \int_{\mathbb{R}^{n}}|\nabla \mathbf{u}|^{2} d x
$$

appears for $n=3$ in Leray's pioneering paper on the Navier-Stokes equations [42]. The constant factor on the right-hand side is sharp. Since one frequently deals with divergence-free fields in hydrodynamics, it is natural to ask whether this restriction can improve the constant in (9.5).

It is shown in [10] that this is the case indeed if $n>2$ and the vector field $\mathbf{u}$ is axisymmetric by proving that the aforementioned constant can be replaced by the (smaller) optimal value

$$
\frac{4}{(n-2)^{2}}\left(1-\frac{8}{(n+2)^{2}}\right)
$$

which, in particular, becomes $68 / 25$ in three dimensions.

However, the following problem remains unsolved. 
Problem 71. Prove or disprove that the constant $4(n-2)^{-2}$ is optimal if $\mathbf{u}$ is an arbitrary divergence-free vector field with components in $C_{0}^{\infty}\left(\mathbb{R}^{n}\right)$.

\section{Miscellaneous Topics}

\subsection{A Modified Riemann's Zeta Function Appearing in a Non-Local Para- bolic Cauchy Problem}

Let $C$ be a unit circle and let $u$ denote a function defined on $C$. Consider the integral operator $A$ :

$$
(A u)(x)=\int_{C} \frac{u(x)-u(y)}{|x-y|}|d y| .
$$

The spectrum of $A$ is described in [79, Section 12.2.2].

Problem 72. Show that the fundamental solution of the Cauchy problem for the operator

$$
\frac{d}{d t}+A, \quad t>0,
$$

which is reminiscent of the modified Riemann zeta function

$$
f(z)=\sum_{n \geq 1} \exp \left(-z \sum_{1 \leq k \leq n} \frac{1}{k}\right),
$$

admits a meromorphic extension to the complex $z=t+i \tau$ plane. Study properties of this extension.

\subsection{Uniqueness Criterion for Analytic Functions with Finite Dirichlet In- tegral}

The following question was raised by Carleson [8]. Suppose that $f$ is analytic in the unit disc $U$ and

$$
\int_{U}\left|f^{\prime}\right|^{p} d A<\infty
$$

for some $p>1$. Let $E$ be a subset of $(-\pi, \pi)$ and suppose that for $\theta \in E$,

$$
f\left(r e^{i \theta}\right) \rightarrow 0 \quad \text { as } r \rightarrow 1
$$

Of what size must $E$ be to force the conclusion that $f$ is identically zero?

In [8], sufficient conditions for a set of zero length which ensure the uniqueness in the case $p=2$ are given. One of the conditions is the positivity of the Riesz potential theoretic capacity $R_{\alpha}$ of a certain positive order $\alpha$.

Maz'ya and Havin [75] described a class of uniqueness sets which is not included into Carleson's. To state our theorem, denote by $E$ a Borel subset of $\partial U$ and let $\Delta$ be a set of non-overlapping open $\operatorname{arcs} \delta \subset \partial U$. Let $|\delta|$ be the length of $\delta$ and put $E_{\delta}:=E \cap \delta$. Suppose $p \in(1,2)$ and

$$
\sum_{\delta \in \Delta}|\delta| \log \frac{|\delta|}{c(\delta)}=-\infty
$$


where $c(\delta):=\operatorname{cap}_{p}\left(E_{\delta}\right)$ is the capacity of $E_{\delta}$ defined by (6.6). Then $E$ is a uniqueness set for $A^{1, p}$, the class of all functions $f$ analytic in $U$ with $f^{\prime} \in L^{p}(U)$.

This theorem is also valid for $p=2$ with a slightly different meaning of $c(\delta)$ due to the peculiarity of dimension two: it is now the cap ${ }_{2}$-capacity of $E_{\delta}$ with respect to the disc $2 d_{\delta}$, where $d_{\delta}$ is a disc contained in $E_{\delta}$. This capacity can be expressed by the logarithmic capacity of $E_{\delta}$ or by its transfinite diameter. The case $(p, \infty)$ can also be included, but it is not interesting. It is not hard to construct a set $E$ satisfying (10.1) whose Riesz capacities $R_{\alpha}(E)$ of any order $\alpha>0$ vanish. Thus our theorem enlarges the class of uniqueness sets for $A^{1,2}$ found in [8].

However, the following problem is still open.

Problem 73. Give a complete characterization of the uniqueness sets for analytic functions in the class $A^{1, p}(U)$.

\subsection{Hybrid Iterative Methods for Solving Boundary Value Problems}

In [34], a mathematical justification of certain new iterative schemes used in solving the Dirichlet and Neumann problems for the Laplace equation is given. These schemes are based on a combination of Green's formula and some numerical methods, FEM, for example, which is applied to some auxiliary mixed boundary value problem on a subset of the original domain.

The proofs in [34] rely upon geometrical requirements on the boundary of the domain of strong convexity type which do not seem natural.

Problem 74. Extend the class of domains with preservation of convergence of the iterative procedures proposed in [34].

\subsection{Asymptotic Optimization of Multi-Structures}

The present section concerns boundary value problems for multi-structures, i.e. domains dependent on small parameters in such a way that the limit region, as parameters tend to zero, consists of subsets of different space dimensions. Asymptotic analysis of physical fields in multi-structures is developed in $[31,77]$ and elsewhere. Direct methods of variational calculus are often ineffective for solving problems of optimal control of multi-structures because of their complicated geometry. However, [77, Remark 4.4] suggests the following promising

Problem 75. Apply algebraic optimization methods to asymptotic approximations of fields in 1D-3D multi-structures.

Open Access. This article is distributed under the terms of the Creative Commons Attribution 4.0 International License (http://creativecommons.org/licenses/by/4. $0 /$ ), which permits unrestricted use, distribution, and reproduction in any medium, provided you give appropriate credit to the original author(s) and the source, provide a link to the Creative Commons license, and indicate if changes were made. 


\section{References}

[1] Adams, D.R., Hedberg, L.I.: Function Spaces and Potential Theory. Springer, Berlin (1996)

[2] Alvarado, R., Brigham, D., Maz'ya, V., Mitrea, M., Zade, E.: On the regularity of domains satisfying a uniform hour-glass condition and a sharp version of the Hopf-Oleinik boundary point principle. J. Math. Sci. (N.Y.) 176(3), 281-360 (2011)

[3] Arnold, V.I.: Local problems of analysis. Vestn. Mosk. Univ. Ser. I Mat. Meh. 25(2), 52-56 (1970). (in Russian)

[4] Arnold, V.I.: Geometrical Methods in the Theory of Ordinary Differential Equations. Grundlehren der Mathematischen Wissenschaften. Springer, Berlin (1983)

[5] Burago, Ju, Maz'ya, V.: Multidimensional potential theory and the solution of the boundary value problems for regions with irregular boundaries. Sem. V.A. Steklov Math. Inst., Leningr. 3, 3-39 (1967)

[6] Baez, J.C., Segal, I.E., Zhou, Z.-F.: The global Goursat problem and scattering for nonlinear wave equations. J. Funct. Anal. 93, 239-269 (1990)

[7] Brennan, J.E.: The integrability of the derivative in conformal mapping. J. Lond. Math. Soc. 2(2), 261-272 (1978)

[8] Carleson, L.: Sets of uniqueness for functions regular in the unit disc. Acta Math. 52, 325-345 (1952)

[9] Cialdea, A., Maz'ya, V.: Semi-Bounded Differential Operators, Contractive Semigroups and Beyond, Operator Theory: Advances and Applications. Birkhäuser/Springer, Cham (2014)

[10] Costin, O., Maz'ya, V.: Sharp Hardy-Leray inequality for axisymmetric divergence-free fields. Calc. Var. Partial Differ. Equ. 32(4), 523-532 (2008)

[11] Dahlberg, B.E.J., Kenig, C.E.: $L^{p}$ estimates for the three-dimensional systems of elastostatics in Lipschitz domains. In: Analysis and Partial Differential Equations, Lecture Notes in Pure and Applied Mathematics, vol. 122, pp. 621-634. Dekker, New York (1990)

[12] Dahlberg, B.E.J., Kenig, C.E., Verchota, G.C.: Boundary value problems for the systems of elastostatics in Lipschitz domains. Duke Math. J. 57(3), 795818 (1988)

[13] Dindoš, M., Maz'ya, V.: $L^{p}$ solvability of the stationary Stokes problem in domains with conical singularity in any dimension. J. Math. Sci. (N.Y.) 170(1), 1-19 (2010)

[14] Eidus, D.: The Cauchy problem for the non-linear filtration equation in a inhomogeneous medium. J. Differ. Equ. 84(2), 309-318 (1990)

[15] Eskin, G.: Index formulas for elliptic boundary value problems in plane domains with corners. Trans. Am. Math. Soc. 314(1), 283-348 (1989)

[16] Frehse, J.: A discontinuous solution of a mildly nonlinear elliptic system. Math. Z. 134, 229-230 (1973)

[17] Fuglede, B.: Extremal length and functional completion. Acta Math. 98, 171219 (1957)

[18] Gelman, I., Mazja, W.: Abschätzungen für Differentialoperatoren im Halbraum. Birkhäuser, Basel (1982) 
[19] Glazman, I.M.: Direct Methods of Qualitative Spectral Analysis of Singular Differential Operators. Translated from Russian by Israel Program for Scientific Translations, Jerusalem, 1965. Daniel Davey, New York (1966)

[20] Gohberg, I., Marcus, A.: Some remarks on topologically equivalent norms. Izv. Mold. Fil. Akad. Nauk SSSR 10(76), 91-94 (1960). (in Russian)

[21] Hardy, G.H., Littlewood, J.E., Polya, G.: Inequalities. Cambridge University Press, Cambridge (1952)

[22] Hörmander, L.: A remark on the characteristic Cauchy problem. J. Funct. Anal. 93(2), 270-277 (1990)

[23] Hörmander, L., Lions, J.L.: Sur la complétion par rapport à une integrale de Dirichlet. Math. Scand. 4, 259-270 (1956)

[24] Jacob, N., Schilling, R.: Function spaces as Dirichlet spaces (about a paper by W. Maz'ya and J. Nagel). Comment on "On equivalent standardization of anisotropic functional spaces $H^{\mu}\left(\mathbb{R}^{n}\right)$ ". Z. Anal. Anwendungen 25(1), 3-28 (2005)

[25] Jaye, B.J., Maz'ya, V., Verbitsky, I.: Existence and regularity of positive solutions of elliptic equations of Schrödinger type. J. Anal. Math. 118(2), 577-621 (2012)

[26] Jaye, B.J., Maz'ya, V., Verbitsky, I.: Quasilinear elliptic equations and weighted Sobolev-Poincaré inequalities with distributional weights. Adv. Math. 232, 513-542 (2013)

[27] Kenig, C.E.: Harmonic analysis technique for second order elliptic boundary value problems. In: CBMS Regional Conference Series in Mathematics, vol. 83 (1994)

[28] Kozlov, V., Maz'ya, V.: Angle singularities of solutions to the Neumann problem for the two-dimensional Riccati's equation. Asymptot. Anal. 19(1), 57-79 (1999)

[29] Kozlov, V., Maz'ya, V.: Spectral properties of operator pencils generated by elliptic boundary value problems in a cone. Funct. Anal. Appl. 22(2), 114-121 (1988)

[30] Kozlov, V., Maz'ya, V.: Asymptotic formula for solutions to elliptic equations near the Lipschitz boundary. Ann. Mat. Pura Appl. 184(2), 185-213 (2005)

[31] Kozlov, V., Maz'ya, V., Movchan, A.: Asymptotic Analysis of Fields in MultiStructures. Clarendon Press, Oxford (1999)

[32] Kozlov, V., Maz'ya, V., Rossmann, J.: Elliptic Boundary Value Problems in Domains with Point Singularities, Mathematical Surveys and Monographs, vol. 52. AMS, Providence (1997)

[33] Kozlov, V., Maz'ya, V., Rossmann, J.: Spectral Problems Associated with Corner Singularities of Solutions to Elliptic Problems, Mathematical Surveys and Monographs, vol. 85. AMS, Providence (2001)

[34] Kozlov, V., Maz'ya, V., Rozin, L.: On certain hybrid iterative methods for solving boundary value problems. SIAM J. Numer. Anal. 31(1), 101-110 (1994)

[35] Král, J.: The Fredholm method in potential theory. Trans. Am. Math. Soc. 125(3), 511-547 (1966)

[36] Kresin, G., Maz'ya, V.: Maximum Principles and Sharp Constants for Solutions of Elliptic and Parabolic Systems, Mathematical Surveys and Monographs, vol. 183. American Mathematical Society, Providence (2012) 
[37] Krol', I., Maz'ya, V.: The absence of the continuity and Hölder continuity of the solutions of quasilinear elliptic equations near a nonregular boundary. Trans. Mosc. Math. Soc. 26, 73-93 (1972)

[38] Kuznetsov, N., Maz'ya, V., Vainberg, B.: Linear Water Waves. A Mathematical Approach. Cambridge University Press, Cambridge (2002)

[39] Landis, E.: On certain properties of solutions to elliptic equations. Dokl. Akad. Nauk SSSR 107(5), 640-643 (1956). (in Russian)

[40] Ladyzhenskaya, O.A.: The Mathematical Theory of Viscous Incompressible Flow. Gordon and Breach, New York (1969)

[41] Lavrentiev, M.M.: On the Cauchy problem for linear elliptic equations of the second order. Dokl. Akad. Nauk SSSR 112, 195-197 (1957). (in Russian)

[42] Leray, J.: Sur le mouvement visqueux emplissant l'espace. Acta Math. 63, 193-248 (1934)

[43] Lewis, J.L.: Approximations of Sobolev functions and related topics. In: Complex Analysis, I (College Park, Md., 1985-86), Lecture Notes in Mathematics, vol. 1275, pp. 223-234. Springer (1987)

[44] Lewis, J.L.: Approximations of Sobolev functions in Jordan domains. Ark. Mat. 25(2), 255-264 (1987)

[45] Littman, W., Stampacchia, G., Weinberger, H.F.: Regular points for elliptic equations with discontinuous coefficients. Ann. Sc. Norm. Pisa (3) 17, 43-79 (1968)

[46] Livshits, M., Maz'ya, V.: Solvability of the two-dimensional Kelvin-Neumann problem for a submerged circular cylinder. Appl. Anal. 64(1-2), 1-5 (1997)

[47] Luo, G., Maz'ya, V.: Pointwise inequalities for elliptic boundary value problems. J. Math. Sci. (N.Y.) 210(4), 391-398 (2015)

[48] Masja, W., Nagel, J.: Über äquivalente Normierung der anisotropen Funktionalräume $H^{\mu}\left(\mathbb{R}^{n}\right)$. Beiträge Anal. 12, 7-17 (1978). (in German)

[49] Maz'ya, V.: The negative spectrum of the $n$-dimensional Schrödinger operator. Sov. Math. Dokl. 3, 808-810 (1962)

[50] Maz'ya, V.: Dirichlet problem for elliptic equations of arbitrary order in unbounded regions. Sov. Math. Dokl. 4, 860-863 (1963)

[51] Maz'ya, V.: On the boundary regularity of solutions of elliptic equations and of a conformal mapping. Dokl. Akad. Nauk SSSR 152, 1297-1300 (1963). (in Russian)

[52] Maz'ya, V.: Regularity at the boundary of solutions of elliptic equations, and conformal mappings. Dokl. Akal. Nauk SSSR 152, 1297-1300 (1963). (in Russian)

[53] Maz'ya, V.: Polyharmonic capacity in the theory of the first boundary value problem. Sib. Mat. Zh. 6, 127-148 (1965). (in Russian)

[54] Maz'ya, V.: On the modulus of continuity of the solutions of the Dirichlet problem near irregular boundary. In: Smirnov, V.I. (ed.) Problems in Mathematical Analysis and Integral Equations; Boundary Problems and Integral Equations, pp. 45-48. Izdat. Leningrad University, Leningrad (1966). (in Russian)

[55] Maz'ya, V.: Behavior near the boundary of solutions of the Dirichlet problem for a second order elliptic equation in divergence form. (in Russian) Mat. Zametki 2(2), 209-220 (1967). English Transl.: Math. Notes 2, 610-617 (1967) 
[56] Maz'ya, V.: The selfadjointness of the Laplace operator. Imbedding theorems and their applications (Proc. Sympos., Baku, 1966), pp. 160-162. Izdat. Nauka, Moscow (1970). (in Russian)

[57] Maz'ya, V.: Classes of sets and measures connected with imbedding theorems. In: Imbedding Theorems and Their Applications (Baku, 1966), pp. 142-159. Nauka, Moscow (1970). (in Russian)

[58] Maz'ya, V.: On the continuity at a boundary point of solutions of quasilinear elliptic equations. Vestnik Leningrad University, 25, 42-55 (1970). [in Russian], English Transl.: Vestnik Leningrad. Univ. Math. 3, 225-242 (1976)

[59] Maz'ya, V.: The degenerate problem with an oblique derivative. Mat. Sb. (N.S.) 87(129), 417-454 (1972). (in Russian)

[60] Maz'ya, V.: On $(p, l)$-capacity, imbedding theorems and the spectrum of a selfadjoint elliptic operator. Izv. Akad. Nauk SSSR, Ser. Mat. 37, 356-385 (1973)

[61] Maz'ya, V.: Certain integral inequalities for functions of many variables. J. Sov. Math. 1, 205-234 (1973)

[62] Maz'ya, V.: Removable singularirties od bounded solutions of quasilinear elliptic equations of any order. J. Sov. Math. 3, 480-492 (1975)

[63] Maz'ya, V.: The index of closure of the operator of the Dirichlet problem in a domain with a nonregular boundary. Problems of Mathematical Analysis, No. 5: Linear and Nonlinear Differential Equations, Differential Operators, 98-121, Izdat. Leningrad University, Leningrad, 1975 (in Russian), English Transl.: J. Soviet Math. 10 (1978), No. 6, 887-908

[64] Maz'ya, V.: On the steady problem of small oscillations of a fluid in the presence of a submerged body. In: Proceedings of the Sobolev Seminar, Novosibirsk: Institute of Mathematics, Sibirian Branch, Acad. Sci. USSR (2), pp. 57-79 (1977). (in Russian)

[65] Maz'ya, V.: On the relationship between the Martin and Euclidean topologies. Sov. Math. Dokl. 18(2), 283-286 (1977)

[66] Maz'ya, V.: Über die Regularität eines Randpunktes für elliptische Differentialgleichungen. In: Linear and Complex Analysis Problem Book, Lecture Notes in Math. vol. 1043, pp. 507-514. Springer (1984)

[67] Maz'ya, V.: The Wiener test for higher order elliptic equations. Duke Math. J. 15(3), 479-512 (2002)

[68] Maz'ya, V.: Lectures on Isoperimetric and Isocapacitary Inequalities in the Theory of Sobolev Spaces. Contemporary Mathematics, vol. 338. American Mathematical Society, Providence (2003)

[69] Maz'ya, V.: A new type of integral equations related to co-area formula (reduction of dimension in multi-dimensional integral equations). J. Funct. Anal. 245(2), 493-504 (2007)

[70] Maz'ya, V.: Estimates for differential operators of vector analysis involving $L^{1}$-norm. J. Eur. Math. Soc. 12(1), 221-240 (2010)

[71] Maz'ya, V.: Sobolev Spaces with Applications to Elliptic Partial Differential Equations. Springer, Berlin (2011)

[72] Maz'ya, V.: Hölder regularity of a boundary point with respect to an elliptic operator of second order. J. Math. Sci. (N.Y.) 196(4), 572-577 (2014) 
[73] Maz'ya, V., Havin, V.: A nonlinear analogue of the Newtonian potential and metric properties of $(p, l)$-capacity. Dokl. Akad. Nauk SSSR 194, 770-773 (1970). (in Russian)

[74] Maz'ja, V., Havin, V.: A nonlinear potential theory. Usp. Mat. Nauk 27(6), 67-138 (1972)

[75] Maz'ya, V., Havin, V.P.: Use of $(p, l)$-capacity in problems of the theory of exceptional sets. Math. USSR Sb. 19, 547-580 (1973)

[76] Maz'ya, V., Havin, V.P.: On the solutions of the Cauchy problem for the Laplace equation (uniqueness, normality, approximations). Trans. Mosc. Math. Soc. 30, 65-117 (1974)

[77] Maz'ya, V., Movchan, A.: Dynamic singular perturbation problems for multistructures. Appl. Stoch. Models Bus. Ind. 16, 249-278 (2000)

[78] Maz'ya, V., Nazarov, S.: The vertex of a cone can be irregular in the Wiener sense for an elliptic equation of the fourth order. Mat. Zametki 39, 24-28 (1986)

[79] Maz'ya, V., Nazarov, S., Plamenevskij, B.: Asymptotic Theory of Elliptic Baundary Value Problems in Singularly Perturbed Domains, vol. I. Birkhäuser, Basel (2000)

[80] Maz'ya, V., Netrusov, Yu.: Some counterexamples for the theory of Sobolev spaces on bad domains. Potential Anal. 4, 47-65 (1995)

[81] Maz'ya, V., Plamenevskii, B.: Properties of solutions of three-dimensional problems of the theory of elasticity and hydrodynamics with isolated singular points. Commun. Math. Phys. 82(2), 99-120 (1981/1982). (in Russian)

[82] Maz'ya, V., Plamenevslii, B.: On the coefficients in the asymptotics of solutions of elliptic boundary value problems in domains with conical points. Am. Math. Soc. Transl. (2) 123, 57-88 (1984)

[83] Maz'ya, V., Poborchi, S.: Differentiable Functions on Bad Domains. World Publishing, New York (1997)

[84] Maz'ya, V., Poborchi, S.: Unique solvability of the integral equation for the simple layer potential on the boundary of a domain with a peak. Vestn. St. Petersb. Univ. Math. 42(2), 120-129 (2009)

[85] Maz'ya, V., Poborchi, S.: Representation of the solution to the Neumann problem for a cuspidal domain by the single layer potential. Vest. St. Petersb. Univ. Math. 42(3), 41-49 (2009)

[86] Maz'ya, V., Poborchi, S.: On solvability of boundary integral equations of potential theory for a multidimensional cusp domain. J. Math. Sci. (N.Y.) 164(3), 403-414 (2010)

[87] Maz'ya, V., Rossmann, J.: Elliptic Equations in Polyhedral Domains, Mathematical Surveys and Monographs, vol. 162. American Mathematical Society, Providence (2010)

[88] Maz'ya, V., Shaposhnikova, T.: Theory of Sobolev Multipliers with Applications to Differential and Integral Operators. Springer, Berlin (2009)

[89] Maz'ya, V., Shaposhnikova, T.: A collection of sharp dilation invariant integral inequalities for differentiable functions. In: Maz'ya, V. (ed.) Sobolev Spaces in Mathematics, I, Int. Math. Ser. (N.Y.), vol. 8, pp. 223-247. Springer, New York (2009)

[90] Maz'ya, V., Shaposhnikova, T.: Higher regularity in the layer potential theory for Lipschitz domains. Indiana Univ. Math. J. 54(1), 99-142 (2005) 
[91] Maz'ya, V., Shubin, M.: Can one see the fundamental frequency of a drum? Lett. Math. Phys. 74(2), 135-151 (2005)

[92] Maz'ya, V., Slutskii, S.: Asymptotic solution to the Dirichlet problem for twodimensional Ricccati's type equation near a corner point. Asymptot. Anal. 39(2), 169-185 (2004)

[93] Maz'ya, V., Soloviev, A.: Boundary Integral Equations on Contours with Peaks, Operator Theory Advances and Applications, vol. 196. Birkhäuser, Basel (2000)

[94] Morrey, C.B.: On the solutions of quasilinear elliptic partial differential equations. Trans. Am. Math. Soc. 43, 126-166 (1938)

[95] Nazarov, S., Plamenevskii, B.: Asymptotic Theory of Elliptic Boundary Value Problems in Singularly Perturbed Domains, vol. I. Birkhäuser, Basel (2000)

[96] Protter, M.H., Weinberger, H.F.: On the spectrum of general second order operators. Bull. Am. Math. Soc. 72, 251-255 (1966)

[97] Pierre, M.: Parabolic capacity and Sobolev spaces. SIAM J. Math. Anal. 14(3), 522-533 (1983)

[98] Putnam, C.R.: On the unboundedness of the essential spectrum. Am. J. Math. 74, 578-586 (1952)

[99] Royden, H.L.: The Growth of a Fundamental Solution of an Elliptic Divergence Structure Equation, 1962 Studies in Mathematical Analysis and Related Topics, pp. 333-340. Stanford University Press, Stanford (1962)

[100] Shen, Zhongwei: Resolvent estimates in $L^{p}$ for the Stokes operator in Lipschitz domains. Arch. Ration. Mech. Anal. 205(2), 395-424 (2012)

[101] Strichartz, R.S.: Multipliers on fractional Sobolev spaces. J. Math. Mech. 16(9), 1031-1060 (1967)

[102] Taylor, M.: Incompressible fluid flow on rough domains, Semigroups of operators: theory and applications, (Newport Beach, CA, 1998). Progr. Nonlinear Differential Equations Appl., vol. 42, pp.320-334. Birkhäuser (2000)

[103] Tolksdorf, P.: On the Dirichlet problem for quasilinear equations in domains with conical boundary points. Commun. Partial Differ. Equ. 8(7), 773-817 (1983)

[104] Toókos, F.: A Wiener-type condition for Hölder continuity for Green's functions. Acta Math. Hung. 111(1-2), 131-155 (2006)

[105] Vainberg, B., Maz'ya, V.: On the plain problem of the motion of a body immersed in a fluid. Trans. Mosc. Math. Soc. 28, 33-55 (1973)

[106] Vainberg, B.R., Maz'ya, V.: The characteristic Cauchy problem for a hyperbolic equation. Trudy Sem. Petrovsk. 7, 101-117 (1981). (in Russian)

[107] Yafaev, D.R.: The Spectrum of the Perturbed Polyharmonic Operator. Problems of Mathematical Physics, No. 5: Spectral Theory, pp. 122-128. Izdat Leningrad University, Leningrad (1971). (in Russian) 


\author{
Vladimir Maz'ya $(\bowtie)$ \\ RUDN University \\ 6 Miklukho-Maklay St \\ Moscow \\ Russia 117198 \\ e-mail: vladimir.mazya@liu.se \\ and \\ Department of Mathematics \\ Linköping University \\ 58183 Linköping \\ Sweden
}

Received: April 4, 2018. 